\title{
A Dynamic Clamp on Every Rig
}

\author{
()iraj S. Desai, ${ }^{*}$ Richard Gray and ${ }^{\circledR}$ Daniel Johnston
}

DOI:http://dx.doi.org/10.1523/ENEURO.0250-17.2017

Center for Learning and Memory and Department of Neuroscience, The University of Texas at Austin, Austin, TX 78712

\begin{abstract}
The dynamic clamp should be a standard part of every cellular electrophysiologist's toolbox. That it is not, even 25 years after its introduction, comes down to three issues: money, the disruption that adding dynamic clamp to an existing electrophysiology rig entails, and the technical prowess required of experimenters. These have been valid and limiting issues in the past, but no longer. Technological advances associated with the so-called maker movement render them moot. We demonstrate this by implementing a fast $(\sim 100 \mathrm{kHz})$ dynamic clamp system using an inexpensive microcontroller (Teensy 3.6). The overall cost of the system is less than USD $\$ 100$, and assembling it requires no prior electronics experience. Modifying it-for example, to add Hodgkin-Huxley-style conductances-requires no prior programming experience. The system works together with existing electrophysiology data acquisition systems (for Macintosh, Windows, and Linux); it does not attempt to supplant them. Moreover, the process of assembling, modifying, and using the system constitutes a useful pedagogical exercise for students and researchers with no background but an interest in electronics and programming. We demonstrate the system's utility by implementing conductances as fast as a transient sodium conductance and as complex as the Ornstein-Uhlenbeck conductances of the "point conductance" model of synaptic background activity.
\end{abstract}

Key words: Dynamic clamp; electrophysiology; patch clamp

\section{Significance Statement}

We describe a system for adding dynamic clamp capability to any existing intracellular electrophysiology rig. Built around a simple microcontroller, the addition is inexpensive ( $<U S D \$ 100)$, can be used in parallel with existing data acquisition systems (and hence entails no disruption of existing experiments), and does not require any technical experience that a typical neuroscientist is unlikely to possess. Its performance is comparable in speed and accuracy to the leading alternatives. This system should make the dynamic clamp method accessible to a wide range of cellular electrophysiologists.

\section{Introduction}

Current clamp and voltage clamp are the standard configurations of cellular electrophysiology. Every trained electrophysiologist is familiar with their properties, and every standard electrophysiological system incorporates

\footnotetext{
Received July 16, 2017; accepted September 11, 2017; First published September 18, 2017.

The authors declare no competing financial interests.

Author contributions: N.S.D., R.G., and D.J. designed research; N.S.D. and R.G. performed research; N.S.D. and R.G. analyzed data; N.S.D., R.G., and D.J. wrote the paper.

NIH MH094839 and NS084473; McKnight Foundation.

Acknowledgments: We are grateful to Elizabeth Arnold, Lauren Hewitt, and Gregory Ordemann for building dynamic clamp systems to test and improve
}

them. And yet there is a third extant and potentially useful configuration: dynamic clamp (a.k.a., conductance clamp; Prinz et al., 2004; Destexhe and Bal, 2009; Prinz and Cudmore, 2011). Introduced independently and concurrently by two different groups (Sharp et al., 1992, 1993;

\footnotetext{
the assembly instructions.

*Correspondence should be addressed to Niraj S. Desai, Center for Learning and Memory, The University of Texas at Austin, Austin, TX 78712. E-mail: desai@utexas.edu.

DOI:http://dx.doi.org/10.1523/ENEURO.0250-17.2017

Copyright (C) 2017 Desai et al.

This is an open-access article distributed under the terms of the Creative Commons Attribution 4.0 International license, which permits unrestricted use, distribution and reproduction in any medium provided that the original work is properly attributed.
} 
Robinson and Kawai, 1993), dynamic clamp is grounded in the idea that the effects that voltage-gated and ligandgated channels have on a neuron's membrane potential can best be understood as changes in conductance rather than in current. The shift in emphasis requires that the electrophysiological system monitor membrane potential and use it, in real time, to calculate what current simulated channels would have passed had they been physically present.

The idea of dynamic clamp is simple, but how to implement it is not. The stumbling block is that dynamic clamp calculations must be done in real time, which is to say faster than any meaningful changes in channel properties or in membrane potential. In mammalian cortex, this means (much) faster than $10 \mathrm{kHz}$. A wide variety of implementations have been proposed since the earliest years, using technically sophisticated manipulations of hardware and software (Dorval et al., 2001; Pinto et al., 2001; Kullmann et al., 2004; Raikov et al., 2004; Desai and Walcott, 2006; Nowotny et al., 2006; Olypher et al., 2006; Milescu et al., 2008; Kemenes et al., 2011; Clausen et al., 2013; Ortega et al., 2014; Biró and Giugliano, 2015; Yang et al., 2015). These efforts have been useful and have had a broad impact, but they have not established dynamic clamp as a part of the standard repertoire of contemporary cellular electrophysiology. The limiting issues have been cost (as much as USD $\$ 6000$, in one case), the requirement in some cases that existing data acquisition systems be replaced or substantially modified rather than merely supplemented, and the technical demands required of users in some cases (e.g., use of a digital signal processing board or facility with $\mathrm{C}++$ or real-time Linux).

These limiting issues have all been valid heretofore, but we would argue that they are no longer. The present decade has witnessed an explosion of projects designed to enable nonengineers and other nonspecialists interested in building tools and other useful objects to make use of modern technological advances (Anderson 2014; Finley 2014; Morozov 2014). The Arduino microcontroller, in the field of embedded electronics, is perhaps the premier example (https://www.arduino.cc). Collectively called the "maker movement," these projects have already had a substantial impact in multiple areas of modern neuroscience, including multielectrode electrophysiology, imaging, behavioral neuroscience, and automated patch clamping (Teikari et al., 2012; Potter et al., 2014; Baden et al., 2015; Desai et al., 2015; Siegle et al., 2017).

In this article, we demonstrate that one product of the maker movement, a microcontroller called the Teensy 3.6 (https://www.pjrc.com), can be used to add dynamic clamp capability to any intracellular electrophysiology rig (whether for patch or sharp electrodes). The addition is cheap (less than USD\$100), can be used in parallel with existing data acquisition systems (and hence entails no disruption of existing experiments), and does not require any technical experience that a typical neuroscience graduate student is unlikely to possess. Indeed, the process of building and using the system will likely teach novices and even moderately experienced researchers some useful things about electronics and programming, as well as initiating them into the potential of other maker movement projects. We demonstrate that the system can handle conductances as fast as a transient HodgkinHuxley-style sodium conductance and as complex as the Ornstein-Uhlenbeck conductances of the "point conductance" model of synaptic background activity (Destexhe et al., 2001).

\section{Methods}

A schematic overview of the system is given in Fig. $1 A$. The portions in black are present on every intracellular electrophysiology rig: an amplifier and a data acquisition (DAQ) board. The amplifier could be a Multiclamp 700B, a Dagan BVC-700A, a HEKA EPC-10, an AM Systems 2400, or any other amplifier that monitors a neuron's membrane potential and injects current into that neuron through a patch or sharp electrode. The DAQ board could be a Molecular Devices Digidata 1500, a HEKA ITC-18, a National Instruments PCle-6343, or any of a huge number of other boards that work with Macintosh, Windows, or Linux operating systems; our dynamic clamp system places no constraint in this regard. We assume that the DAQ board is controlled on the host computer by a DAQ system suitable for intracellular electrophysiology. The system could be one of the commercial systems on the market (e.g., Molecular Devices pClamp 10 or AxoGraph), or it could be open source (e.g., Janelia's Wavesurfer) or homemade. The point is simply that the electrophysiology rig already includes the components necessary to patch or impale neurons and record current clamp data.

Into this existing, working configuration, we insert the portion of Fig. $1 A$ depicted in red. It consists of a Teensy 3.6 microcontroller and associated electronics. We chose the microcontroller because, compared with other devices of its class and in its price range, it is fast ( $180 \mathrm{MHz}$ clock speed), has substantial memory (256 kB RAM), and has a floating point unit (more on this in Discussion). The Teensy is responsible in our system for performing all the dynamic clamp calculations. It determines, moment by moment, what current a voltage- or ligand-gated conductance would pass were it physically present and adds this to the current that the existing DAQ system has been instructed to inject (e.g., a family of current steps). That is, the existing DAQ system continues to perform all the standard current clamp (or voltage clamp) functions. The Teensy system simply adds a dynamic clamp component: it adds the current from simulated conductances to the current that the existing current clamp system specifies.

For the Teensy to do this, some electronic additions are required (Fig. 1B): (1) a power supply, (2) circuitry to map the voltage output of the intracellular amplifier (typically $\pm 9 \mathrm{~V}$ ) representing the neuron's membrane potential to the voltages the Teensy can read $(0-3.3 \mathrm{~V})$, (3) electrical connections to and from the Teensy, (4) circuitry to transform the Teensy's output (0-3.3 V) into a voltage the intracellular amplifier can correctly interpret (typically \pm 9 V) as a current (in pA) to be injected into the neuron, and (5) circuitry to sum the dynamic clamp currents specified 
A

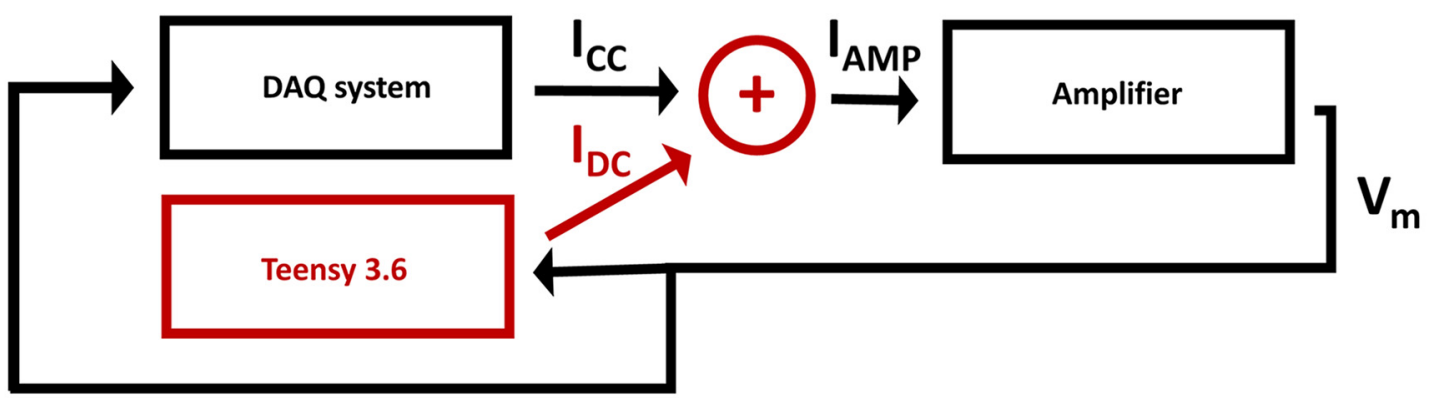

B

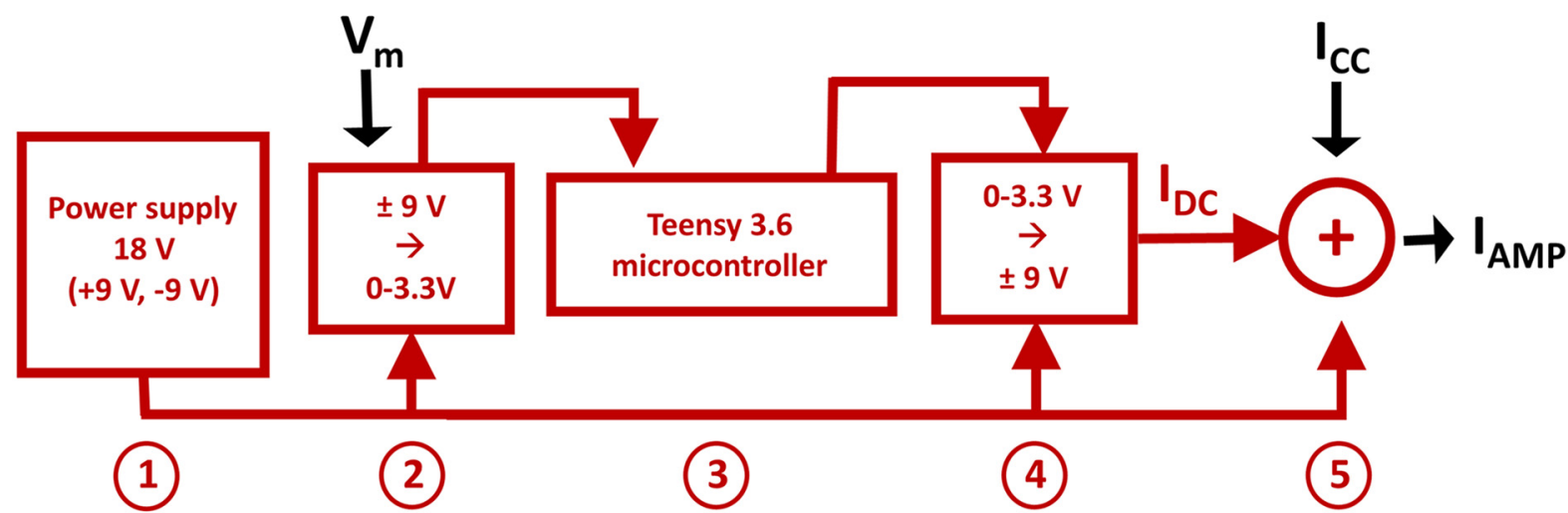

C
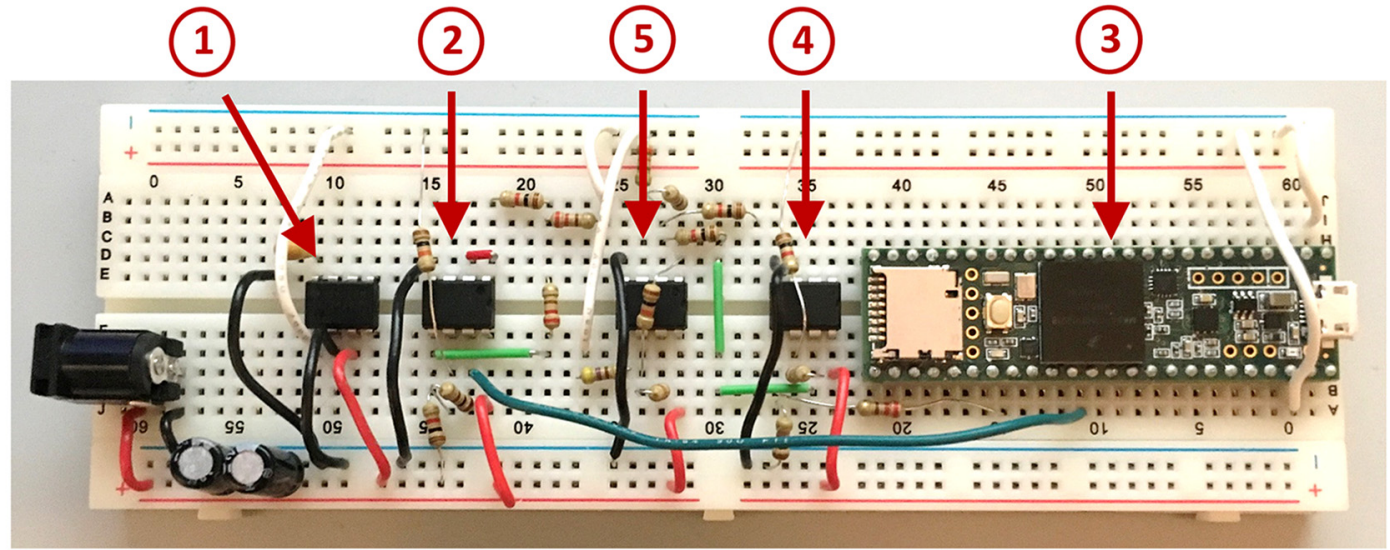

Figure 1. Schematic representations of the system. $\boldsymbol{A}$, The Teensy 3.6 microcontroller and its associated electronics (red) are added to an existing system (black) consisting of an intracellular amplifier and a data acquisition (DAQ) system. The amplifier sends the membrane potential $V_{m}$ to both the DAQ system and the Teensy system. The DAQ system, which could (for example) be comprised of a Digidata 1500 and pClamp 10 software, records $V_{m}$ to disk as usual and specifies whatever current $\left(\mathrm{l}_{\mathrm{CC}}\right)$ it would inject in a standard current-clamp configuration. The microcontroller uses $V_{m}$ to calculate what current $\left(\mathrm{l}_{\mathrm{DC}}\right)$ the dynamic clamp conductances would have passed had they been physically present. The sum of these two currents, $\mathrm{I}_{\mathrm{AMP}}=\mathrm{I}_{\mathrm{CC}}+\mathrm{I}_{\mathrm{DC}}$, is sent to the command input of the amplifier to be injected into the neuron. $\boldsymbol{B}$, The Teensy system consists of five parts: (1) a power supply $18 \mathrm{~V}$, which is broken up into a positive voltage (9 V) and a negative voltage ( $-9 \mathrm{~V})$ to power the other circuits and to provide both positive and negative rails; (2) a differential amplifier circuit that maps the output of the intracellular amplifier, which will be in the range $\pm 9 \mathrm{~V}$, onto the range $0-3.3 \mathrm{~V}$ that the Teensy can read; (3) the Teensy controller itself; (4) a second differential amplifier circuit that maps the output of the Teensy, which will be in the range $0-3.3 \mathrm{~V}$, onto the range $\pm 9 \mathrm{~V}$ the intracellular amplifier expects at its command input; and (5) a summing circuit that adds the voltage commands representing $\mathrm{I}_{\mathrm{CC}}$ and $\mathrm{I}_{\mathrm{DC}}$. A voltage representing the sum $\mathrm{I}_{\mathrm{AMP}}$ is sent to the intracellular amplifier and thereafter injected into the neuron. $\boldsymbol{C}$, The entire system can be built on a standard solderless breadboard. The five parts of the system are indicated by the arrows. All of the components (resistors, capacitors, ICs, wires, and microcontroller) can be secured simply by pushing their wires into the breadboard holes; no soldering is required. A detailed, step-by-step description of how to assemble the five parts of the system on a breadboard is available in the Extended Data 1 ("Assembling the system on a solderless breadboard"). 
by the Teensy and the current clamp currents specified by the DAQ system. We explain these five additions, all of which can be built on a single solderless breadboard (Fig. $1 C)$, in the five sections that follow.

After that, we discuss the software that controls the Teensy. Although the Teensy 3.6 is not a microcontroller of the Arduino line (www.arduino.cc), it is very similar and can be programmed using the (open-source) Arduino integrated development environment (IDE). We provide, in the online material, the code we used to program various simulated conductances; these serve as examples for users who might wish to program different simulated conductances. We also discuss code written in the (opensource) Processing language (www.processing.org) to change dynamic clamp parameters "on the fly" (i.e., during a recording). Processing is a useful language because it is very simple and because, like Arduino, its code can be used without modification on all three major operating systems (Macintosh, Windows, and Linux). We end the methods section by discussing how to calibrate the electronic components of the dynamic clamp system to ensure best performance.

Source code, a parts list, photographs, and step-bystep instructions are included in the Extended Data 1. Postpublication updates will be available at a website we have created for this purpose (dynamicclamp.com), with software archived at the public repository Github (https:// github.com/nsdesai/dynamic_clamp)

\section{Power supply}

The power supply serves two purposes: it provides power for the operational amplifiers ("op-amps") of the other circuits, and it provides the positive (negative) reference voltages the other circuits use to shift up (down) the voltages sent to (from) the Teensy microcontroller. For both these purposes, we require a positive voltage (approximately $9 \mathrm{~V}$ ) and a negative voltage (approximately $-9 \mathrm{~V}$ ).

The simplest power source suitable for both purposes is an 18-V DC wall adaptor (colloquially called a "wall wart"). Such an adaptor typically terminates in a barrel plug that can be plugged into a barrel connector. (We provide a full parts list, including links to supplier webpages, in the Extended Data 1, so readers can see for themselves what all the parts look like.) This power source cannot work alone because it is positive only, whereas we wish to have both positive and negative voltages. More precisely, we wish to break up the $18 \mathrm{~V}$ into one rail at 9 $\mathrm{V}$, one rail at $-9 \mathrm{~V}$, with a ground (called a "virtual ground") right at the halfway point.

Circuits that perform this function are called "rail splitters." In principle, a purely passive rail splitter circuitessentially a voltage divider-would suffice here, but in this design we opted for an op-amp circuit, because it minimizes asymmetry between the positive and negative rails and buffers the power supply from the downstream circuits. (Keeping one part of the system from interfering with other parts-"buffering"-is a general principle of electronic design.) The op-amp circuit we chose is a ubiquitous integrated circuit (IC) from Texas Instruments
(TLE2426). Fig. $2 A$ shows schematically how the IC is connected.

In the Extended Data 1, we describe and show (with pictures) how the circuit looks when the parts are physically connected on a solderless breadboard. The breadboard has four power rails. One rail is marked $9 \mathrm{~V}$ and another is marked $-9 \mathrm{~V}$. The downstream circuits use these two rails to power their own op-amps and as reference points for $9 \mathrm{~V}$ and $-9 \mathrm{~V}$. A third rail on the breadboard is connected to the virtual ground of the rail splitter. Every other voltage in this system will be referenced to this ground rail.

\section{Amplifier output to microcontroller input}

An intracellular electrophysiology amplifier in current clamp mode monitors a neuron's membrane potential and outputs a signal representing this value. For real neurons, the membrane potential will be in a range no wider than -90 to $90 \mathrm{mV}$. The representative output will depend on the gain of the amplifier, but for the typical settings of commonly used amplifiers the corresponding range will be -9 to $9 \mathrm{~V}$. This is too broad a range for the analog inputs of the Teensy microcontroller (or other controllers of this class), which are limited to 0-3.3 V.

To map $\pm 9 \mathrm{~V}$ from the amplifier onto $0-3.3 \mathrm{~V}$ to the microcontroller, we employ three distinct elements (Fig. $2 B$ ). The first is a follower circuit. It takes an input ( $\pm 9 \mathrm{~V})$ and simply sends out an identical output $( \pm 9 \mathrm{~V})$. The follower's purpose is to separate-buffer-the input from the output, to keep them from interfering with each other. The second is a voltage divider that transforms the voltage from the amplifier $( \pm 9 \mathrm{~V})$ onto a more limited range (approximately $\pm 1.6 \mathrm{mV}$; the precise numbers depend on the precise resistor values chosen). The third is a differential amplifier that adds (approximately) $1.6 \mathrm{~V}$ to shift the output of the second element into the range $0-3.2 \mathrm{~V}$, which roughly matches the dynamic range of the Teensy's analog-to-digital (ADC) input.

One can calculate the relationship between the input $\left(V_{I N}\right)$ to this three-element circuit and its output $\left(V_{\text {OUT }}\right)$ by assuming that all the resistor values and power supply voltages are exact and that all the op-amps are ideal (Senturia and Wedlock, 1975), as follows:

$$
\begin{aligned}
V_{\text {OUT }}=(1+ & \left.\frac{R_{6}}{R_{5}}\right)\left[\frac{R_{4} R_{2}}{\left(R_{3}+R_{4}\right)\left(R_{1}+R_{2}\right)} V_{I N}\right. \\
& \left.+\frac{R_{3}}{\left(R_{3}+R_{4}\right)} V_{+}\right] .
\end{aligned}
$$

In this equation, $V_{+}$is the voltage of the positive power rail $(9 \mathrm{~V})$. Note that the relationship between $V_{I N}$ and $V_{\text {OUT }}$ is linear. We constructed this circuit on a breadboard using resistor values between $100 \Omega$ and $22 \mathrm{k} \Omega$ (specified in the figure caption) and an IC that contains two op-amps (LM358n). Testing the breadboard circuit, we found that the empirical relationship between $V_{I N}$ and $V_{\text {OUT }}$ was indeed strictly linear (see Calibration), but that the numerical values of the slope and intercept were somewhat different from what the exact equation would predict (by $\sim 2 \%$ ). This discrepancy resulted from imperfections in the (inex- 


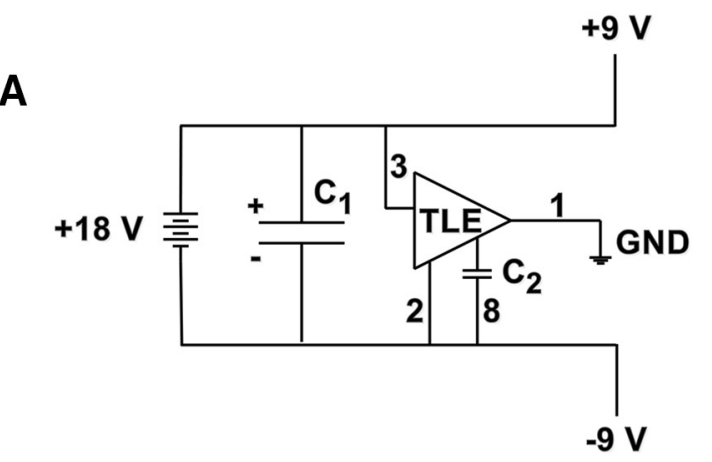

B

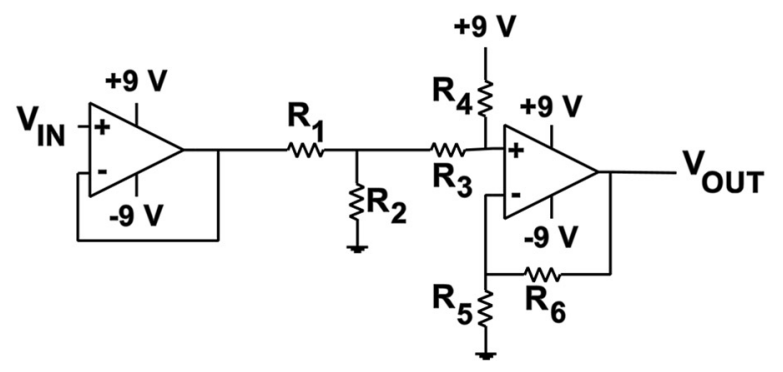

C

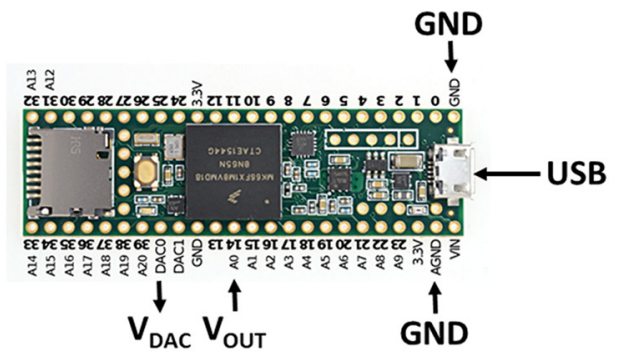

D

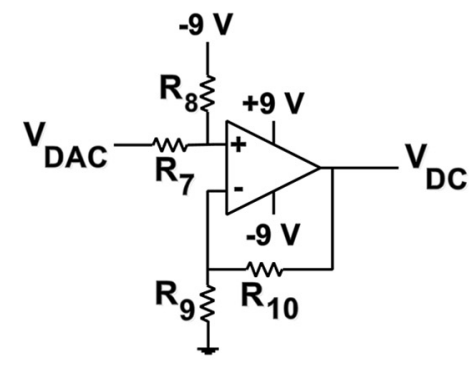

E

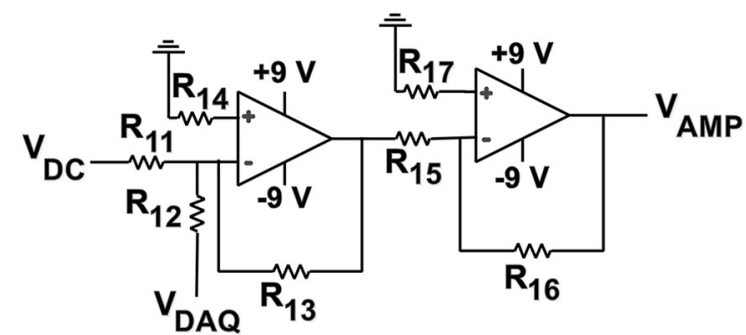

Figure 2. Breadboard electronics. The five parts of the system illustrated in Fig. $1 B$ are shown schematically. $\boldsymbol{A}$, Rail splitter power supply. An 18-V power supply (wall adaptor) is split by a TLE 2426 rail splitter IC into $9 \mathrm{~V},-9 \mathrm{~V}$, and ground. The capactors
Figure 2. continued

are $\mathrm{C}_{1}=200 \mu \mathrm{F}$ and $\mathrm{C}_{2}=1 \mu \mathrm{F}$. The dark numbers refer to the pins of the TLE 2426 IC. B, Follower, voltage divider, and differential amplifier circuits to map the range -9 to $9 \mathrm{~V}$ onto the range 0 to $3.3 \mathrm{~V}$. The resistor values are $R_{1}=2200 \Omega, R_{2}=470 \Omega$, $R_{3}=4700 \Omega, R_{4}=22,000 \Omega, R_{5}=10,000 \Omega$, and $R_{6}=100 \Omega$. C, Connections to the Teensy 3.6 microcontroller. The output of the previous circuit is fed to pin $\mathrm{AO}$, and the output of pin DACO is fed to the next circuit. $\boldsymbol{D}$, Differential amplifier circuit to transform the output of the microcontroller $(0-3.3 \mathrm{~V}$, representing the dynamic clamp current) into a range ( $\pm 9 \mathrm{~V}$ ) expected by the intracellular amplifier. The resistor values are $R_{7}=4700 \Omega, R_{8}=$ $22,000 \Omega, R_{9}=10,000 \Omega$, and $R_{10}=10,000 \Omega$. $\boldsymbol{E}$, Summing amplifier. The voltage command from the DAQ board (representing the current clamp's specified current) is added to the voltage command from the Teensy microcontroller. The sum is sent to the command input of the intracellular amplifier. Resistors $R_{11}$, $R_{12}, R_{13}, R_{15}$, and $R_{16}$ are $10,000 \Omega$; resistor $R_{14}$ is $3300 \Omega$; and $R_{17}$ is $4700 \Omega$.

pensive) electronic components we chose and the nonideal behavior of the op-amps of the LM358n chip.

Fortunately, the discrepancy can be corrected in software, without having to substitute better (and more expensive) electronic components. As explained in Calibration, this can be done by measuring $V_{\text {OUT }}$ values in response to a range of known $V_{I N}$ values. The numbers can be fitted to a straight line and the resulting slope and intercept used instead of the calculated slope and intercept.

\section{Microcontroller connections}

The output of the three-element circuit (now 0-3.2 V) is fed to an ADC input on the Teensy microcontroller (Fig. $2 C)$. The Teensy 3.6 has 25 ADC inputs; our default software simply selects the first of these (A0, pin 14), but any can be used. The Teensy analog ground should be connected to the virtual ground defined by the rail splitter circuit. The Teensy has two digital-to-analog (DAC) outputs; our default software uses the first of these (A21).

\section{Microcontroller output to amplifier input}

The output of the Teensy DAC will be a voltage between 0 and $3.3 \mathrm{~V}$, but most amplifiers in current-clamp mode expect command voltages between -9 and $9 \mathrm{~V}$, with negative voltages representing hyperpolarizing current injections and positive voltages representing depolarizing current injections. Mapping 0-3.3 V onto the range $\pm 9 \mathrm{~V}$ is the inverse of the problem we faced earlier, and its solution is similar but inverted. We use a differential amplifier both to shift $0-3.3 \mathrm{~V}$ down to the range $\pm 1.65 \mathrm{~V}$ and to amplify the result (Fig. 2D).

Assuming perfect, ideal components, we can calculate the relationship between the input supplied by the Teensy $\left(V_{D A C}\right)$ and the output of the circuit $\left(V_{D C}\right.$, representing the dynamic clamp command signal; Senturia and Wedlock, 1975):

$$
V_{D C}=\left(1+\frac{R_{10}}{R_{9}}\right)\left[\frac{R_{8}}{\left(R_{7}+R_{8}\right)} V_{D A C}+\frac{R_{7}}{\left(R_{7}+R_{8}\right)} V_{-}\right]
$$


Here, $V_{-}$is the voltage of the negative power rail $(-9 \mathrm{~V})$. Again, the relationship between input $\left(V_{D A C}\right)$ and output ( $V_{D C}$ ) is linear. And again, when we constructed the circuit on a breadboard, we found that, although the empirical relationship between $V_{D A C}$ and $V_{D C}$ was linear, it was not strictly given by the calculated formula (off by $\sim 2 \%$ ). This discrepancy too can be resolved in software (see Calibration).

\section{Summing circuit}

The fifth and last electronic circuit is designed to sum the dynamic clamp command voltage $V_{D C}$ and the current clamp command voltage from the DAQ system $V_{D A Q}$. Summing voltages is a common electronics task, and we perform it in a standard fashion: an inverting amplifier that sums the two voltages but switches their polarity, followed by a second inverting amplifier that switches the polarity back (Fig. 2E).

\section{Software}

\section{Arduino IDE}

There are multiple ways of programming the Teensy 3.6, including simply using the $C$ language, but the most sensible way to do so is through the Arduino IDE (https:// www.arduino.cc/en/Main/Software). Arduino has emerged over the last $5 \mathrm{y}$ as the microcontroller of choice of the maker movement, including nearly all the neuroscience-related projects (Potter et al., 2014; Baden et al., 2015; Desai et al., 2015; Siegle et al., 2017). The Arduino IDE and its associated language retain the essential syntax of $\mathrm{C}$ while making the process of interfacing with a microcontroller straightforward. Although Teensy is not part of the Arduino line of microcontrollers, a Teensy-specific add-on to the Arduino IDE exists and allows one to use the IDE and most of its libraries (https://www.pjrc.com/teensy/teensyduino.html). Detailed installation and use instructions are included in the Extended Data 1.

We wrote our dynamic clamp software using the Arduino IDE. The code is contained in the Extended Data 1 folder dynamic_clamp and the main file is called dynamic_clamp.ino. Opening the latter opens not only the main file but also its associated files, which appear in separate tabs. Each tabbed file contains the code for a specific conductance. The main file is structured in three parts: global variables, a setup function, and a loop function. The global variables are self-explanatory (variables needed by all and therefore accessible to all functions); the setup function is run once when the program is uploaded to the board and does things like initialize serial communication between the host computer and the Teensy board; the loop function is run at every time step: it calls each of the conductance-specific tabbed files to get the current specified by that conductance.

In the example software, we coded five separate conductances: (1) a simple shunt conductance, (2) a hyperpolarization-activated cyclic nucleotide-gated (HCN) conductance (Gasparini et al., 2004), (3) a fast, transient sodium conductance (Johnston and Wu, 1994), (4) an excitatory postsynaptic conductance (EPSC; Compte et al., 2000), and (5) "high conductance state" synaptic background conductances (Destexhe et al., 2001, 2003).
The first is simple. The second and third are HodgkinHuxley conductances with one and four gates, respectively. The fourth is a synaptic conductance that is triggered by a transistor-transistor logic $(T T L)$ pulse sent by the DAQ board to the Teensy microcontroller. The fifth is comprised of two conductances-one excitatory, one inhibitory-generated by Ornstein-Uhlenbeck (OU) processes. Our example code demonstrates how to numerically integrate the stochastic OU equations and how to generate the Gaussian random numbers the OU processes require (Marsaglia and Bray, 1964). Together, these five examples span the range of conductances users are likely to wish to use, and this code is meant to provide templates from which users can create other conductances. As a further aid, in the Extended Data 1, we also describe step-by-step how to add a potassium M conductance (Fransen et al., 2002).

\section{Processing}

When the Arduino program is uploaded to the Teensy microcontroller, all of the dynamic clamp conductances are initialized to zero. They can be changed to nonzero values while the program is running. That is, the dynamic clamp conductances can be changed on the fly during a given recording.

The simplest way for the host computer to tell the microcontroller to modify the dynamic clamp conductances is through the USB port that connects them. In our default Arduino software, the microcontroller constantly checks for a serial communication from the host computer and changes the conductance values as soon as it arrives. Unfortunately, the Arduino IDE itself has no good way of sending real-time communication from the host computer to the microcontroller. Fortunately, many other programs do. One called Processing (www.processing. org) is especially well suited for this purpose: it is an open-source environment with a simple syntax (based on Java and thus possessing a family resemblance to the $\mathrm{C}$-like Arduino) that has a huge user base and is platform independent (Windows, Macintosh, or Linux).

In the Extended Data 1, we include a Processing sketch (called processing_control.pde) that creates a graphical user interface (GUI) through which users can change the values of the maximal conductances (in nanoSiemens) of the five conductances of our default software. The GUI also allows users to modify the diffusion constants (in square nanoSiemens per millisecond) of the OU processes. All the parameters can be modified by moving the sliders in the GUI and pressing the "upload" button.

But there is nothing unique about Processing. Users are free to use any software they wish in order, for example, to couple their data acquisition and dynamic clamp software more tightly. To emphasize this point, we also include Matlab software (Windows, Macintosh, or Linux) in the Extended Data 1 that does the same things as the Processing sketch.

\section{Calibration}

Electronic components are typically specified with some margin of error (e.g., the specified resistance or capacitance will be good only to within 1\%) and no op- 
A

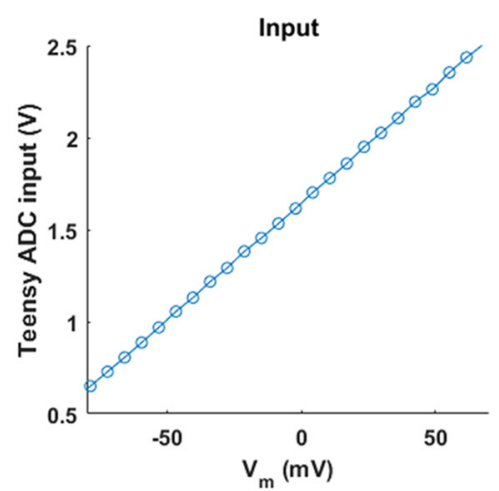

Output

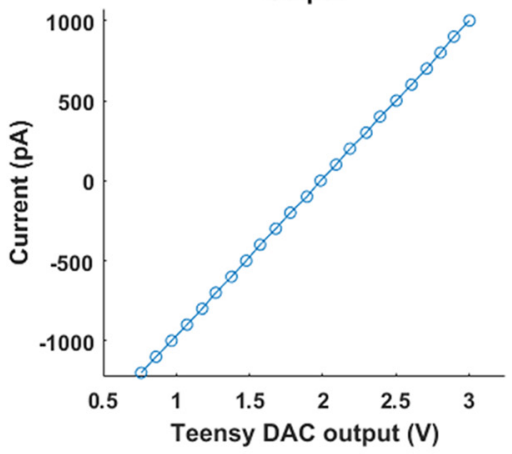

B

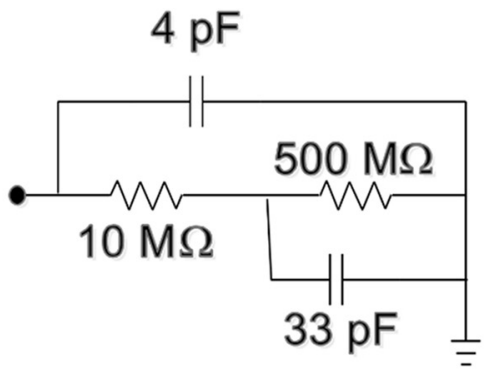

C

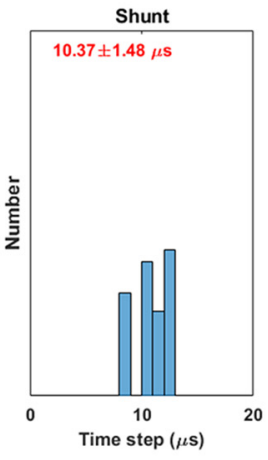

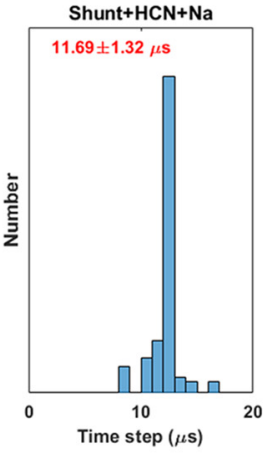
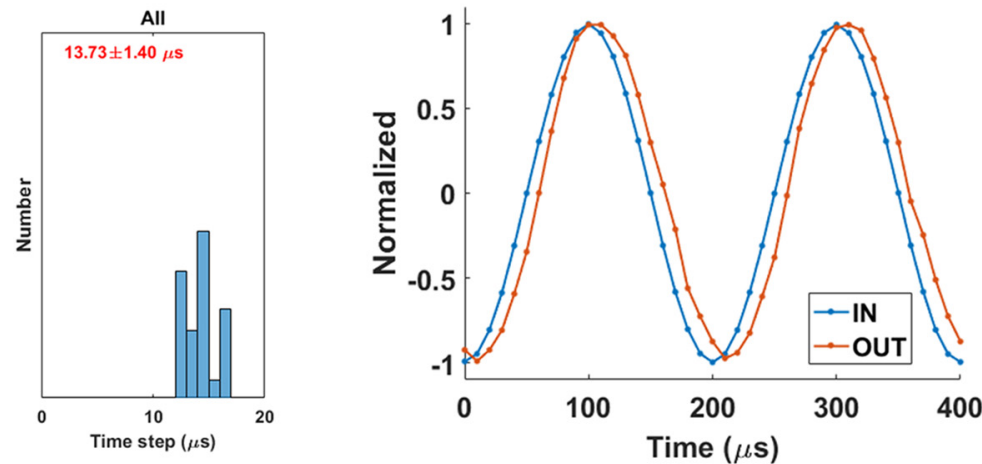

Figure 3. Calibrating and testing the system. $\boldsymbol{A}$, Although the electronic components were not ideal, the input-output characteristics of the system were highly linear. Left, the voltages measured by the microcontroller's analog input in response to a range of membrane potentials. Right, the currents injected into a model cell in response to a range of voltages sent out by the microcontroller's analog output. $\boldsymbol{B}$, To test the system, a model cell was attached to the intracellular amplifier's headstage. Shown is the model's equivalent circuit. $\boldsymbol{C}$, The system's speed approaches $100 \mathrm{kHz}$ and depends only weakly on the number of conductances being simulated. We recorded the durations of 51,200 time steps (at microsecond resolution) for each of three dynamic clamp configurations: shunt conductance only; shunt, HCN, and sodium conductances together; and shunt, HCN, sodium, OrnsteinUhlenbeck, and EPSC conductances all together. Shown in the three figures at left are the resulting histograms; the number at the top of each is mean \pm SD. The temporal jitter in all cases was 1-2 $\mu \mathrm{s}$. To check the temporal latency, sinusoidal voltages $(5 \mathrm{kHz})$ were fed to the system's input (replacing $V_{I N}$ of Fig. $\left.2 B\right)$ and the resulting outgoing current commands $\left(V_{A M P}\right.$ of Fig. 2E) were measured for a shunt conductance $(2 \mathrm{nS})$. Both the input signal and the output signal were sampled at $100 \mathrm{kHz}$. The latency between input and output was roughly $10 \mu \mathrm{s}$.

amp or other active component behaves ideally. This means that the slope and intercept values for the electronic circuits calculated above will be not quite correct. Empirically, using the components specified in the parts list, we find that "not quite correct" means "not good enough."

Fortunately, the input-output functions of the circuits in question (Fig. 2B,D) are strictly linear (Fig. 3A). We determined this by directly measuring the output of the two circuits when they were subjected to a range of input voltages. In principle, users could do the same thing we did in that figure: use the DAQ board to send a voltage directly to the circuit input (Fig. $3 A$, left) or to measure the circuit output directly (Fig. $3 A$, right). But a slightly simpler method exists: attach a model cell to the headstage of the amplifier, measure the responses to a variety of current/ voltage commands, and use these together with the model cell's known architecture (arrangement of resistances and capacitances) to calculate the calibration parameters. The model cell could be as simple as a single resistor, but in Fig. $3 B$ we used a Patch-1U model cell ("cell mode") attached to a Multiclamp 700B amplifier (Molecular Devices). In cell mode, this model cell incorporates two resistors, representing input resistance and series resistance, and two capacitors, representing membrane capacitance and stray capacitance (due to the glass electrode; Fig. 3B).

In the Extended Data 1, we include a Processing sketch and step-by-step instructions for calibrating the system using a model cell attached to an amplifier.

\section{Testing}

We tested the dynamic clamp system mainly using a Windows 10 computer, Multiclamp 700B amplifier (Molecular Devices), PCle-6343 data acquisition board (National Instruments), Patch-1U model cell (Molecular Devices), and custom Matlab (The Mathworks) data acquisition software. To check that the system was indeed independent of operating system and equipment type, we also tested it on a rig with a Mac OS X computer, an Axopatch 200B amplifier (Molecular Devices), an ITC-18 
data acquisition board (HEKA Instruments), and custom Igor Pro (Wavemetrics) data acquisition software.

Where indicated, we replaced the model cell in our tests with whole-cell patch-clamp recordings from mouse layer $2 / 3$ or 5 pyramidal neurons. The mice were C57BL/6 males (6-8 wks old, Jackson Laboratory). Brain slices containing medial prefrontal cortex were prepared using standard procedures. Shortly after receiving a lethal dose of ketamine/xylazine, mice were perfused transcardially with an ice-cold solution containing (in $\mathrm{mM}$ ): $2.5 \mathrm{KCl}, 1.25$ $\mathrm{NaH}_{2} \mathrm{PO}_{4}, 25 \mathrm{NaHCO}_{3}, 0.5 \mathrm{CaCl}_{2}, 7 \mathrm{MgCl}_{2}, 7$ dextrose, 205 sucrose, 1.3 ascorbate, and 3 sodium pyruvate (bubbled with $95 \% \mathrm{O}_{2} / 5 \% \mathrm{CO}_{2}$ to maintain $\mathrm{pH}$ at $~ 7.4$ ). Brains were removed, and a vibratome was used to make 300$\mu \mathrm{m}$-thick coronal sections. Slices were cut in the same ice-cold saline used for perfusion and then were held for 30 min at $35^{\circ} \mathrm{C}$ in a chamber filled with artificial CSF containing (in mM): $125 \mathrm{NaCl}, 2.5 \mathrm{KCl}, 1.25 \mathrm{NaH}_{2} \mathrm{PO}_{4}, 25$ $\mathrm{NaHCO}_{3}, 2 \mathrm{CaCl}_{2}, 2 \mathrm{MgCl}_{2}, 25$ dextrose, 1.3 ascorbate, and 3 sodium pyruvate (bubbled with $95 \% \mathrm{O}_{2} / 5 \% \mathrm{CO}_{2}$ ). Thereafter, they were maintained at room temperature in the same solution. Patch-clamp recordings were obtained under visual guidance at $35^{\circ} \mathrm{C}$ using patch electrodes (3-7 $\mathrm{M} \Omega$ ) filled with an internal solution containing (in mM): 125 K-gluconate, $10 \mathrm{KCl}, 4 \mathrm{NaCl}, 10 \mathrm{HEPES}, 4 \mathrm{Mg}$-ATP, 0.3 Tris-GTP, and 7 phosphocreatine $(\mathrm{pH} 7.4$ at physiologic temperatures). All animal procedures were approved by the Institutional Animal Care and Use Committee of the University of Texas at Austin and were in accordance with National Institutes of Health guidelines.

\section{Software accessibility}

The software for the system is available, together with other useful materials (a parts list, assembly and use instructions, photographs), as Extended Data 1. These are also available at a website we have created for this purpose (dynamicclamp.com), with software archived by the public repository Github (https://github.com/nsdesai/dynamic_clamp). Any postpublication improvements to the hardware or software will be available at these sites.

\section{Results}

We validated the dynamic clamp system in two distinct but complementary ways: (1) with a model cell (Fig. 3B) attached to the amplifier headstage, and (2) with wholecell patch-clamp recordings from layer $2 / 3$ or 5 pyramidal neurons in slices of mouse prefrontal cortex (6-8 wks old). The first was our principal method because it provided a steady baseline against which the effects of added (simulated) conductance could be reliably contrasted. The second was useful because it more closely matched the experimental configuration in which potential users of this system are likely to be interested.

\section{Timing}

One question is important in both cases: how fast is the dynamic clamp? For a single simulated conductance, the answer approaches $100 \mathrm{kHz}$, with a jitter of $<2 \mu \mathrm{s}$. We determined this by measuring the distribution of time steps for different dynamic clamp configurations (Fig. 3C, three leftmost panels). Moreover, the time per cycle does not grow linearly with the number of simulated conductances, because most of the time cost (analog read + analog write) is fixed and common for all conductances. So, for example, simulating shunt, $\mathrm{HCN}$, and sodium conductances at the same time can be done at better than $80 \mathrm{kHz}$ (Fig. 3C, second from left). In keeping with these numbers, the latency between membrane potential $V_{m}$ and the dynamic clamp current was $\sim 10 \mu \mathrm{s}$ (Fig. 3C, right).

In principle, the system could be sped up by using less averaging when reading from an analog input or using higher-quality op-amps (with a larger bandwidth and slew rate). However, we found-and show in what followsthat the system as described and constructed can handle even the most challenging conductance (i.e., transient sodium) without difficulty. We discuss the potential speed improvements in Discussion.

\section{Model cell: shunt conductance}

The simplest intrinsic conductance a neuron might possess is a shunt conductance, which is a constant conductance. The current it passes is the product of its amplitude and the driving force (the difference between the membrane potential and the conductance's reversal potential): $I=-g_{\text {shunt }}\left(V_{m}-E_{\text {rev }}\right)$. In Fig. 4, we added a shunt conductance to the model cell of Fig. 3B; we used the amplifier's built-in bridge balance and capacitance compensation circuitry to minimize the effects of series resistance and pipette capacitance. From the diagram, the equivalent circuit, after balance and compensation, should have been $500 \mathrm{M} \Omega$ in parallel with $33 \mathrm{pF}$. However, the components used to make up the model cell are far from ideal (see the Molecular Devices page on the model cell's precision: http://bit.ly/2qHavi1). By injecting a variety of time-varying currents, we found that the model cell's parameters were better fitted by resistance and capacitance values of $507.7 \mathrm{M} \Omega$ and $35.9 \mathrm{pF}$. We used these numbers to check the precision of our dynamic clamp currents.

In Fig. 4, we injected a family of current steps (-50 to 50 $\mathrm{pA}$ ) into the model cell. The model was simply a resistor in parallel with a capacitor (an RC circuit). We therefore expected and found that the voltage responses to the steps were exponential growth and decay. Adding $5 \mathrm{nS}$ of shunt conductance preserved the basic shape of the responses-all the shunt conductance does is decrease the value of $\mathrm{R}$ - but it reduced the steady-state deflections and made the responses faster. To check the system's behavior quantitatively, we used a National Instruments DAQ board (50 kHz, 16 bit) to directly measure the dynamic clamp current emitted by the system and to compare it to the current that should have been passed given the recorded membrane potential had the system worked perfectly. These numbers (Fig. 4, upper right, measured versus numerical) were in good agreement. The inset shows a histogram of the deviation (error) between the measured and numerical currents during the steps. The absolute value of the error averaged $0.2 \pm 0.2$ $\mathrm{pA}$ (mean $\pm \mathrm{SD}$ ). Moreover, when we varied the amplitude of the shunt conductance, the measured input resistances 

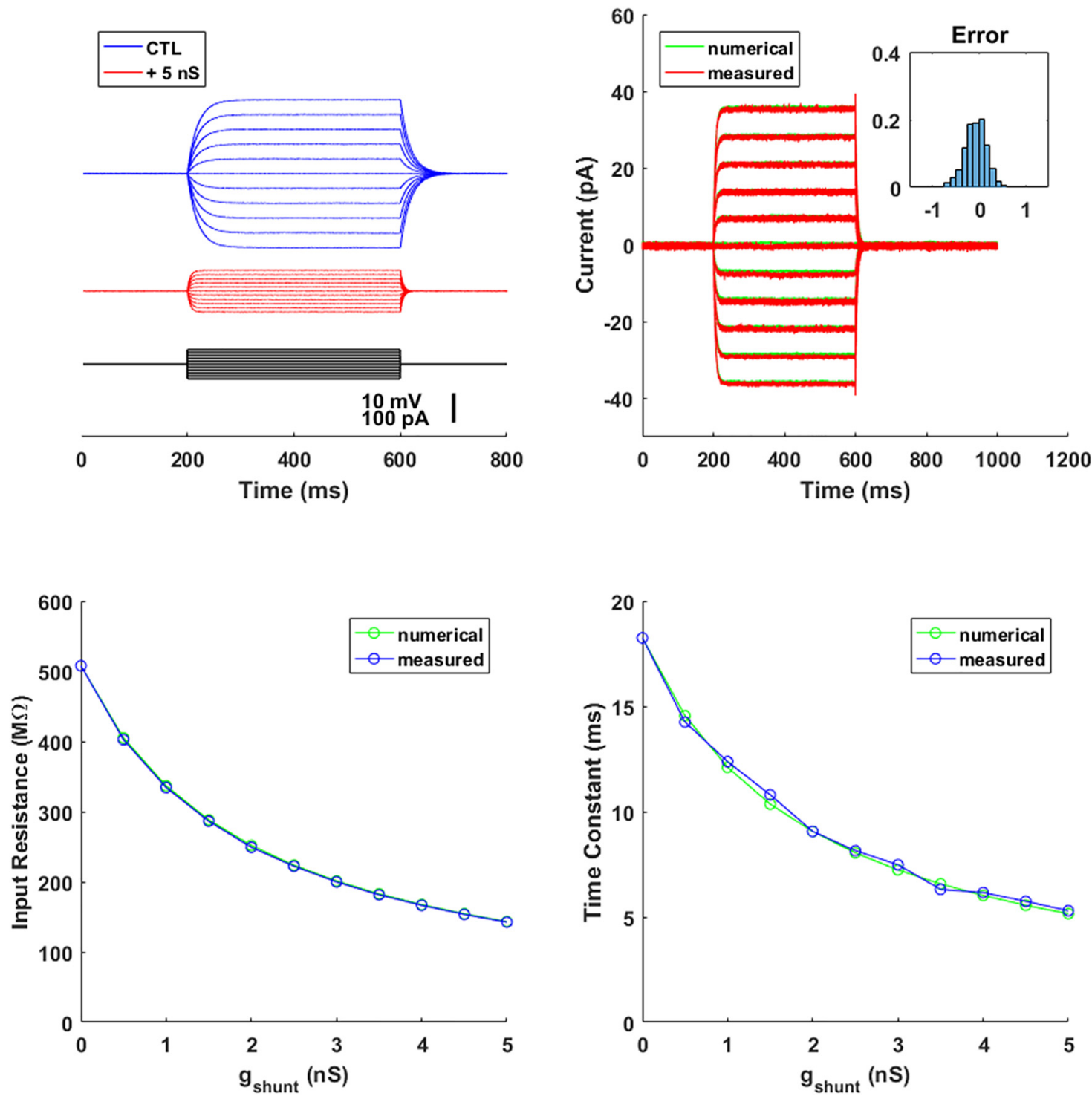

Figure 4. Shunt conductance. Adding $5 \mathrm{nS}$ of shunt conductance to the model cell reduced and quickened the voltage deflections to a family of current steps (upper left). The shunt currents added by the dynamic clamp system (measured directly by the DAQ board) closely matched what they should have been given the recorded membrane potentials (numerical; upper right). The inset shows a probability histogram of the difference (error) between the measured and numerical currents. Varying the amplitude of the shunt conductance affected the input resistance and time constant (measured) as expected given the numerical values of the model cell resistance and the shunt conductance amplitudes (numerical; lower panels).

(lower left) and time constants (lower right, estimated by fitting single exponentials to the voltage responses to current steps) closely matched the correct values determined by numerical calculation. The average difference was $<2 \%$, and in no case was the difference $>5 \%$.

\section{Model cell: HCN conductance}

One step up in complexity from a shunt conductance is an intrinsic conductance with a single activation gate. Several such conductances are important physiologically, including delayed-rectifier potassium and the HCN conductance. Here we focus on the HCN conductance. It activates but does not inactivate. What makes the conductance unusual is that the proportion of activated channels is increased by hyperpolarization (Fig. $5 A$ ) and that the reversal potential sits near the base of the activation curve. This configuration imbues the HCN conductance with interesting physiologic properties (Biel et al., 2009). The dynamics of the HCN conductance can be modeled by a single differential equation:

$$
\frac{d s(t)}{d t}=-\left[s(t)-s_{i n f}\left(V_{m}\right)\right] / \tau_{s}\left(V_{m}\right),
$$

where $s(t)$ represents the fraction of open HCN channels at any moment in time $t$, and $s_{\text {inf }}(V)$ and $\tau_{s}(V)$ are the voltage-dependent steady-state value and time constant, respectively (Fig. 5A; Gasparini et al., 2004). Our dynamic clamp system integrated this equation using the forward Euler method with a time step of $\sim 10 \mu \mathrm{s}$. 
A

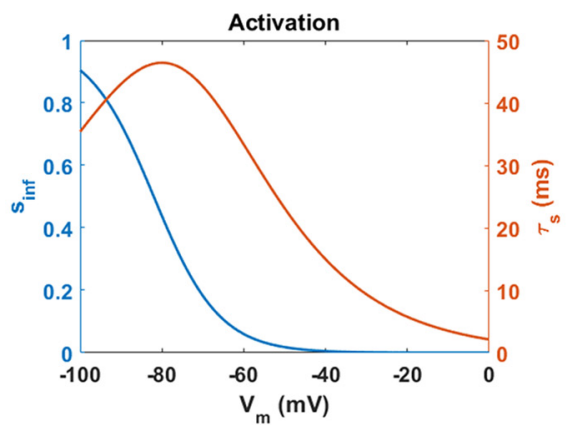

B

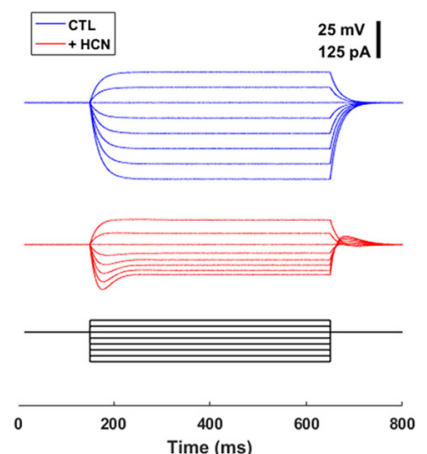

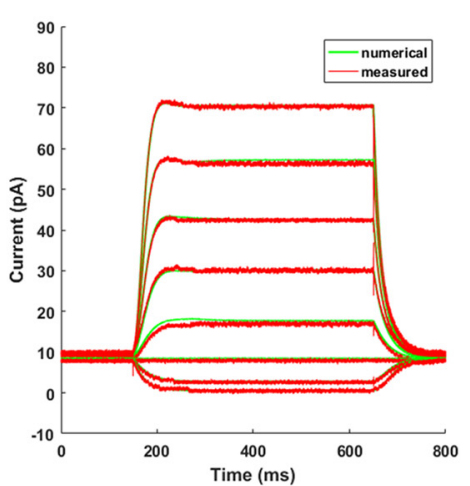

D

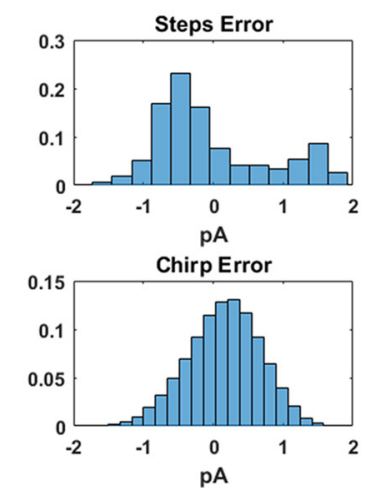

C
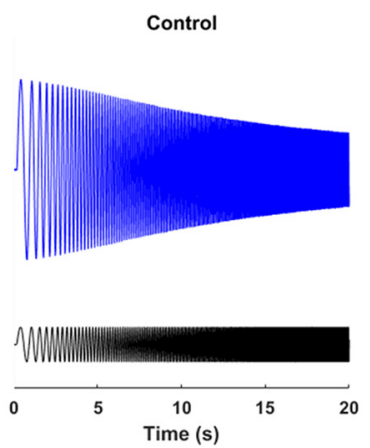

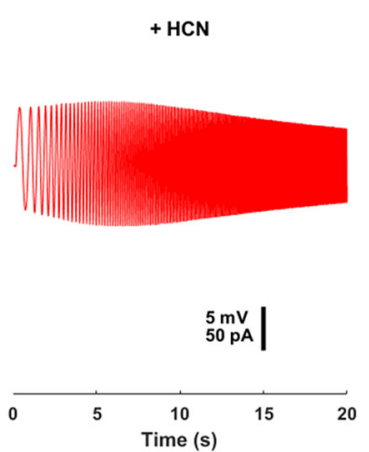

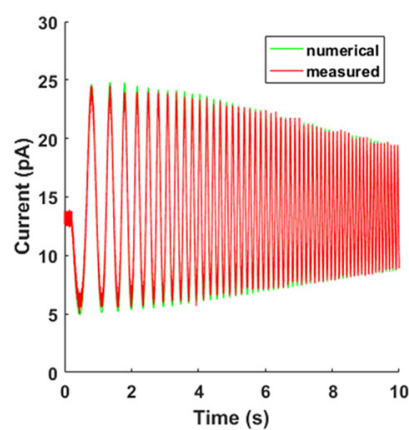

Figure 5. HCN conductance. $\boldsymbol{A}$, The conductance was modeled by a single-activation gate that had a steady-state value $s_{\text {inf }}(V)$ and a time constant $\tau_{s}(V)$. B , Current steps $(-100$ to $40 \mathrm{pA})$ were injected into a model cell without $(\mathrm{CTL})$ and with $(+\mathrm{HCN})$ the addition of $2 \mathrm{nS} \mathrm{HCN}$ conductance. Note that adding the simulated conductance resulted in the appearance of a sag potential (left). At right, the currents injected by the dynamic clamp system (measured, directly by the DAQ board) for the eight steps are plotted together with the result of numerically integrating the Hodgkin-Huxley equation using the fourth-order Runge-Kutta method (numerical). The top histogram ("steps error") of $\boldsymbol{D}$ shows how good the agreement between the measured and predicted (numerical) currents was. $\boldsymbol{C}$, The model cell (Fig. $3 B$ ) is essentially an "RC circuit." In response to a time-varying input, it acts like a low-pass filter. This can be seen (left) by its response to a chirp stimulus (black); the voltage deflection steadily decreases as the frequency increases. Addition of 4 nS HCN conductance transforms the system into a bandpass filter, with a resonant frequency. Again, the agreement between the current injected by the dynamic clamp system (measured) and the expected current given by numerical integration of the Hodgkin-Huxley equations (numerical) was excellent. $\boldsymbol{D}$, Histograms of the error between the measured and expected currents for step currents (top) and the chirp current (bottom).

How well it did this is shown in Fig. 5B,C. Two important physiologic signatures of an $\mathrm{HCN}$ conductance are sag and resonance (Biel et al., 2009). Adding a simulated HCN conductance to the model cell introduced a sag potential (Fig. 5B) when the model was subjected to hyperpolarizing current steps. (The baseline potential of the model cell here and below was set at a resting potential of $-70 \mathrm{mV}$.) The HCN conductance also effectively added an inductance to the circuit (termed a "phenomenological inductance" by Narayanan and Johnston, 2008). This transformed the equivalent circuit of the model cell from an RC circuit to an RLC circuit: it transformed the cell from a low-pass filter into a bandpass filter, with a distinct resonance frequency (Fig. $5 C)$.

More important than these qualitative effects was that the simulated currents were quantitatively correct. We directly measured the currents emitted by the dynamic clamp system and compared them to the currents specified by precise numerical integration (fourth-order Runge-Kutta, 10- $\mu$ s time step) of the differential equation. The agreement was very good (Fig. $5 B-D$ ); for both step currents and oscillating (chirp) currents, the average error (absolute value, mean $\pm \mathrm{SD}$ ) was $<1 \mathrm{pA}$ (steps: $0.7 \pm 0.5$ $\mathrm{pA}$; oscillating: $0.4 \pm 0.3 \mathrm{pA}$ ), and in no case was the absolute error ever $>2 \mathrm{pA}$.

\section{Model cell: sodium conductance}

The classic Hodgkin-Huxley formulation of the sodium conductance involves three identical and independent activation gates $m(t)$ and a single inactivation gate $h(t)$ (Johnston and Wu, 1994). The behavior of each is determined by a differential equation that involves voltagedependent functions:

$$
\begin{gathered}
\frac{d m(t)}{d t}=-\left[m(t)-m_{\text {inf }}\left(V_{m}\right)\right] / \tau_{m}\left(V_{m}\right), \\
\frac{d h(t)}{d t}=-\left[h(t)-h_{\text {inf }}\left(V_{m}\right)\right] / \tau_{h}\left(V_{m}\right) .
\end{gathered}
$$

The voltage-dependent functions are shown in Fig. $6 A$. The sodium current is given by $I=-g_{\mathrm{Na}} m(t)^{3} h(t)\left(V_{m}-\right.$ 
A
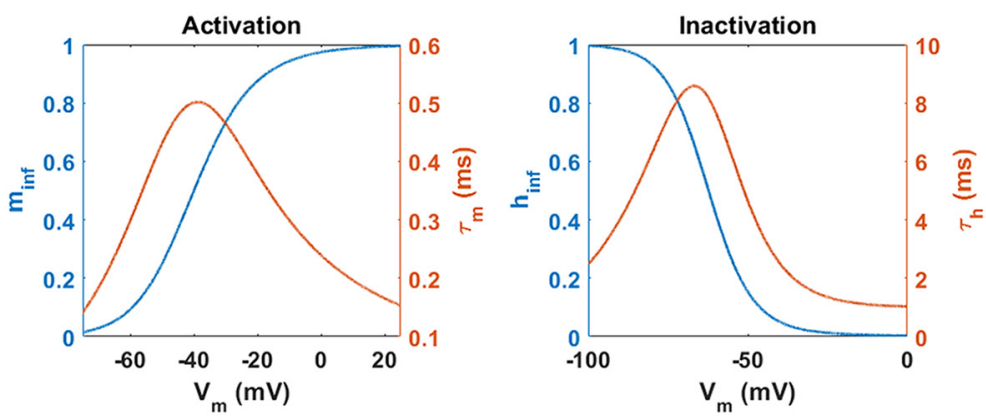

C
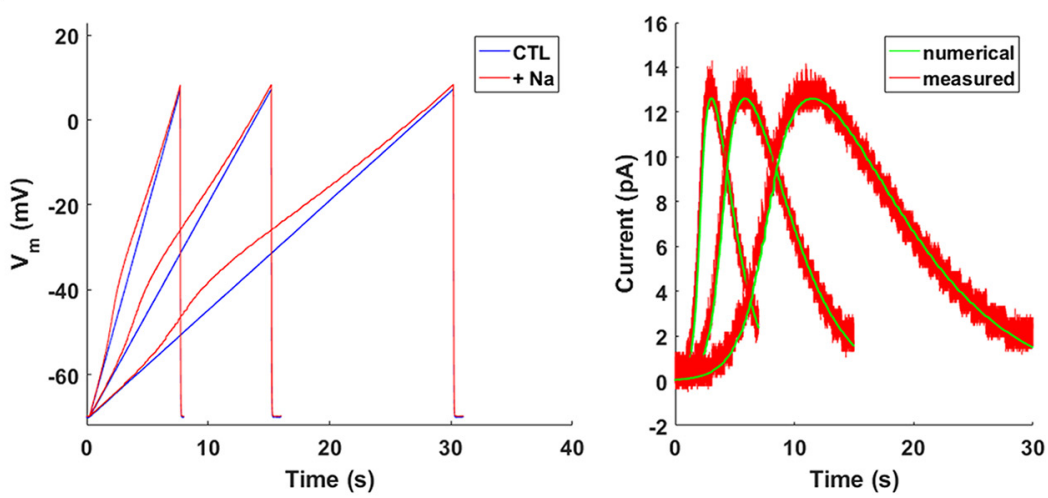

D
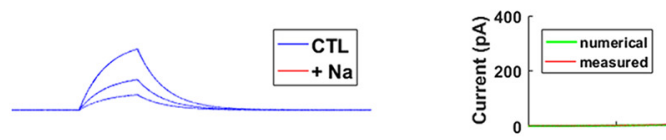

$25 \mathrm{pA}$

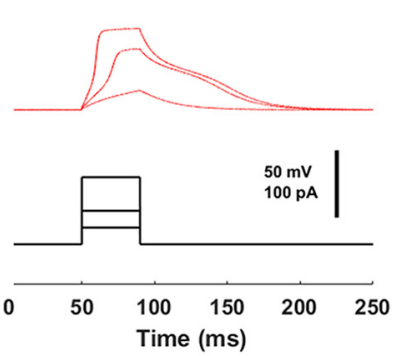

B
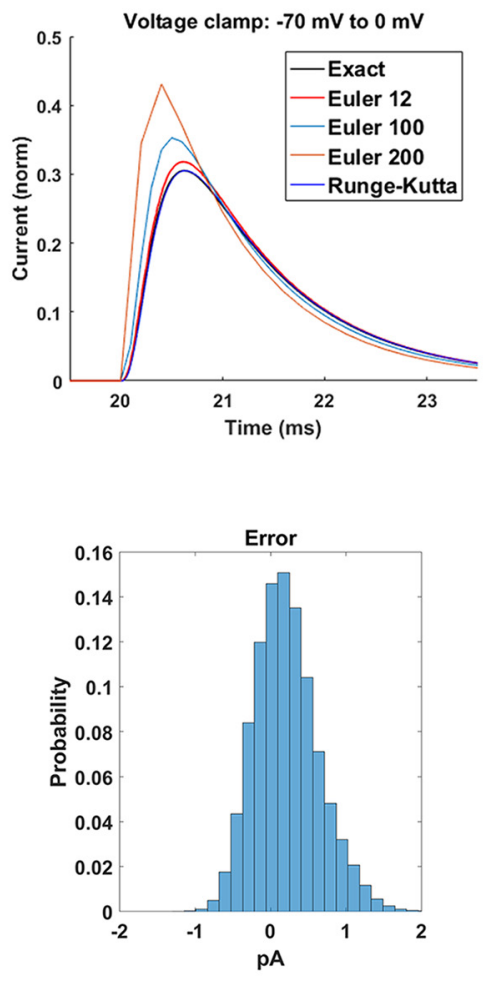
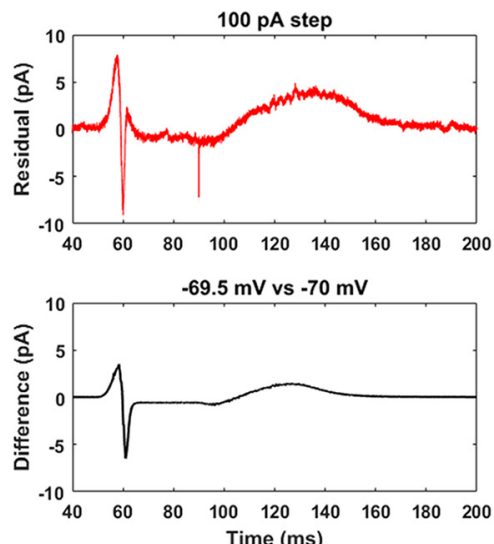

Figure 6. Sodium conductance. $\boldsymbol{A}$, The conductance was modeled using both an activation gate $m\left(V_{m}, t\right)$ and an inactivation gate $h\left(V_{m}, t\right)$. The steady-state and kinetic values of the two gates are plotted. The total sodium current was given by $g_{N a} m^{3} h\left(V_{m}-E_{N a}\right)$, where $g_{N a}$ is the maximal sodium conductance and $E_{\mathrm{Na}}$ is the sodium reversal potential (50 mV). $\boldsymbol{B}$, Comparison of different numerical integration methods. In a simulation, the voltage was stepped instantaneously from $-70 \mathrm{mV}$ to $0 \mathrm{mV}$ at a time $t=20 \mathrm{~ms}$. The resulting (inward) sodium current was calculated using the forward Euler method (time step 200, 100, or $12 \mu \mathrm{s}$ ), the fourth-order Runge-Kutta method (time step $10 \mu \mathrm{s}$ ), or an exact analytical calculation (exact). For the Euler simulation of $12 \mu \mathrm{s}$, the time step was also jittered by $2 \mu \mathrm{s}$ (standard deviation). $\boldsymbol{C}$, At left, the response of the model cell to slow current ramps $(5,10$, and $20 \mathrm{pA} / \mathrm{s})$ is plotted in the absence $(\mathrm{CTL})$ and presence $(+\mathrm{Na})$ of an added sodium conductance $\left(g_{\mathrm{Na}}=20 \mathrm{nS}\right)$. In the middle, the close agreement between the sodium currents produced by the dynamic clamp system (measured) and those expected from precise numerical integration of the Hodgkin-Huxley equations (numerical) is demonstrated. At right is a histogram of the error (difference) between the measured and expected currents. $\boldsymbol{D}$, The model cell was subjected to brief current steps. Without a sodium conductance, the responses showed pure exponential growth, as expected of an RC circuit. With a sodium conductance $\left(g_{\mathrm{Na}}=80 \mathrm{nS}\right)$, the responses showed nonlinear behavior above a threshold $(>25 \mathrm{pA})$. The sodium currents (measured directly and expected from numerical integration) are plotted in the middle. Not only do the currents agree with each other, but they show a striking threshold behavior. At right (top), the error (difference) between the measured current and the expected current is plotted for the largest current step (100 pA). The shape of the error is consistent with what would be expected from a small offset in the baseline membrane potential. At right (bottom) is the difference between the fourth-order Runge-Kutta (time step $10 \mu \mathrm{s}$ ) estimate of the current for a baseline of $-70 \mathrm{mV}$ and the estimate for a baseline of $-69.5 \mathrm{mV}$. 
$E_{\text {rev }}$ ), where $g_{N a}$ is the maximal sodium conductance and $E_{\text {rev }}=50 \mathrm{mV}$ is the sodium reversal potential. Integrating these equations in real time is more challenging than in the $\mathrm{HCN}$ case not only because there are now two equations rather than one, but also because the characteristic time associated with the activation gate $\tau_{m}(V)$ is very short $(<0.5 \mathrm{~ms})$. This sets the required time scale.

The microcontroller integrated these equations using the forward Euler method with a time step of $<12 \mu \mathrm{s}$. To check that this was satisfactory, we simulated a voltage clamp experiment in which the model cell was stepped instantly from -70 to $0 \mathrm{mV}$. The sodium current elicited by such a step can be written down exactly. We compared the exact solution to those calculated using various numerical methods (Fig. 6B). As expected, the fourth-order Runge-Kutta method, which is widely used in modeling studies because of its stability and precision (Bettencourt et al., 2008), matched the exact solution almost perfectly. How well the forward Euler method did depended on the time step: time steps longer than $200 \mu$ s gave unstable solutions; those between 25 and $200 \mu$ s were stable but imprecise; but those $<25 \mu$ s were generally satisfactory. In particular, forward Euler with a time step of $12 \mu \mathrm{s}$ and a jitter of $2 \mu \mathrm{s}$, which matches the microcontroller's performance, differed from the exact solution only near the peak of the sodium current and then only by a few percentage points (Fig. 6B, compare black and red traces). This result suggests that, with the possible exception of some fine details of spike shape (Bettencourt et al., 2008), forward Euler may be used to simulate Hodgkin-Huxleystyle sodium conductances as long as the time step is $<25 \mu \mathrm{s}$, which is the case here.

We tested the dynamic clamp system's performance by using two types of stimuli (Fig. $6 C, D$ ): slow current ramps (5-20 pA/s) and brief current steps (0-100 pA, $40 \mathrm{~ms})$. In response to the ramps, the simulated sodium current activated and inactivated as expected (Fig. 6C, left); the match between the measured currents and those predicted by a precise numerical integration (fourth-order Runge-Kutta, 10- $\mu$ s time step) was very good-the absolute value of the error averaged $0.4 \pm 0.3 \mathrm{pA}$ (Fig. $6 C$, right). Even more striking was the response to brief current steps: these exhibited threshold behavior (Fig. $6 D$, left). Small currents $(<25 \mathrm{pA})$ moved the model cell's potential only a small distance from its baseline of -70 $\mathrm{mV}$, thus eliciting minimal sodium current. But larger currents $(>50 \mathrm{pA})$ resulted in sharp, spike-like bursts of sodium current. In the latter cases, the deviation between the measured current and the current expected from a precise numerical integration could be as large as $9 \mathrm{pA}$ (Fig. $6 D$, right, top). This error was still small relative to the size of the underlying currents $(>300 \mathrm{pA})$, and it likely originated from small imperfections in the patch clamp amplifier settings. Sodium conductance is very sensitive to such deviations. For example, if the baseline potential had been off by $0.5 \mathrm{mV}$ because the model cell's starting potential was not precisely $-70 \mathrm{mV}$, that alone would have resulted in an error of the same shape and magnitude as the one we measured (Fig. $6 D$, right, bottom), without any contribution from the dynamic clamp system.

\section{Model cell: EPSCs}

Synaptic inputs to central neurons are mostly, although not exclusively, mediated by chemical synapses. These usually exhibit a rise time that is much faster than the decay time. There are several distinct ways of modeling the time course of synaptic currents, such as alpha functions and the difference of exponentials. Here we illustrate a particularly useful and general two-stage kinetic scheme (Destexhe et al., 1994; Compte et al., 2000; Walcott et al., 2011). We use it to model AMPA currents, but it is straightforward to modify it to model other types of synaptic currents. The kinetic scheme involves two variables:

$$
\begin{gathered}
\frac{d x(t)}{d t}=-\frac{x(t)}{\tau_{x}}+\sum_{i} \delta\left(t-t_{i}\right), \\
\frac{d s(t)}{d t}=-\frac{s(t)}{\tau_{s}}+\alpha_{s} x(t)[1-s(t)],
\end{gathered}
$$

where $\alpha_{s}$ is a constant determining saturation properties; $\tau_{s}$ and $\tau_{x}$ are time constants controlling decay and rise times, respectively; $\delta(t)$ is the Dirac delta function; and $\left\{t_{i}\right\}$ are the presynaptic spike times. The resulting current is given by $I=-g_{s y n} s(t)\left[V_{m}(t)-E_{\text {rev }}\right]$, where $E_{\text {rev }}$ is the synaptic reversal potential. To model AMPA currents, we set $E_{\text {rev }}=0 \mathrm{mV}, \tau_{s}=10 \mathrm{~ms}, \tau_{x}=1 \mathrm{~ms}$, and $\alpha_{s}=$ $1 \mathrm{~ms}^{-1}$.

The presynaptic spike times $\left\{t_{i}\right\}$ were determined by $T T L$ triggers sent by the DAQ system. We sent triggers at rates of 10,20, and $50 \mathrm{~Hz}$ (Fig. $7 A$ ). The higher frequencies showed evidence of synaptic summation. As was true of intrinsic conductances, the agreement between the measured dynamic clamp currents and numerical estimates of ideal behavior was excellent (Fig. 7B). This agreement can be quantified by considering a histogram of the errors, computed as the difference between the measured and expected currents, with the latter derived from a precise numerical integration of the differential equations (Fig. 7C). The average absolute error across all frequencies was $0.2 \pm 0.2 \mathrm{pA}$ (mean $\pm \mathrm{SD}$ ); the largest errors, which occurred at the peaks of the EPSCs, were $<1.5 \mathrm{pA}$.

\section{Model cell: synaptic background activity}

Most electrophysiological studies of neuronal properties have been conducted in vitro, using preparations such as brain slices and cell cultures, where the electrical and chemical background can be tightly controlled. However, neurons in vivo confront a rather different environment: they receive a continuous barrage of excitatory and inhibitory synaptic inputs, even in the absence of sensory or motor stimulation; a significant portion of total conductance is contributed by synaptic conductances rather than intrinsic ones; neurons are depolarized above rest by a much as $10 \mathrm{mV}$; and "resting" membrane potential fluctuates by as much as $5 \mathrm{mV}$ (Steriade, 2001; Destexhe et al., 2003). A useful dynamic clamp tool to explore differences between in vitro and in vivo was contributed by Destexhe et al. (2001), who demonstrated that the electrical portion of synaptic background activity might be 
A

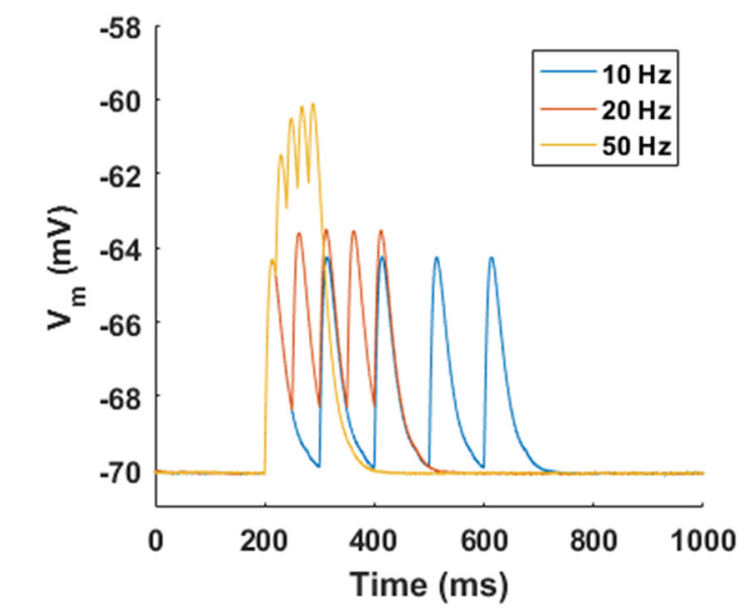

C

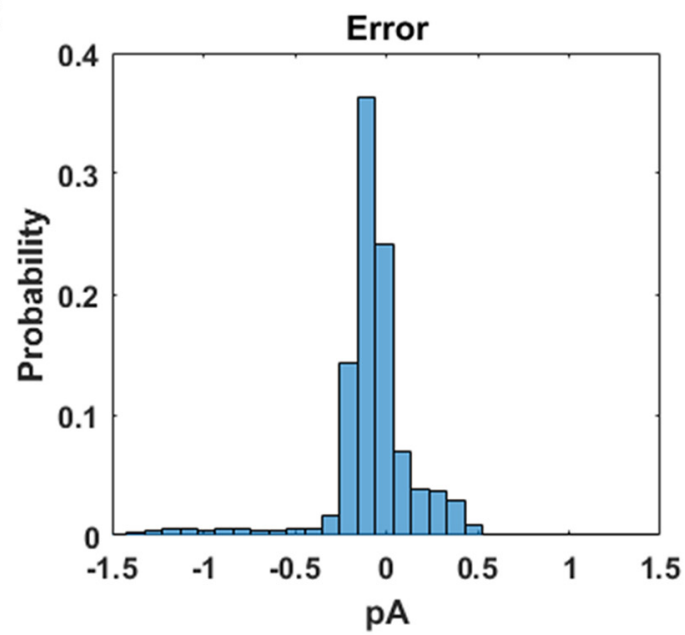

B
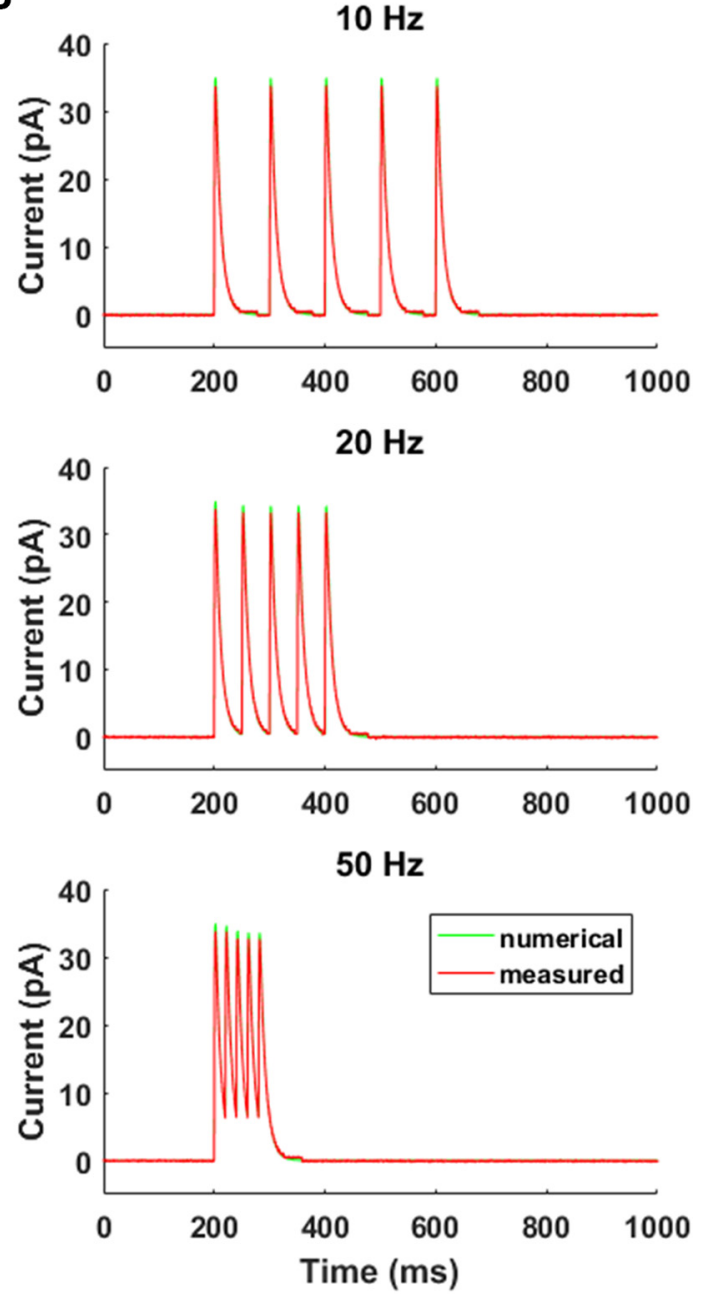

Figure 7. Excitatory postsynaptic currents. $\boldsymbol{A}$, EPSCs were triggered at fixed times with stimulation frequencies of 10,20, and $50 \mathrm{~Hz}$. Note how the potentials summate for the higher frequencies. $\boldsymbol{B}$, The agreement between the currents injected by the dynamic clamp system (measured) and the expected currents given by precise Runge-Kutta numerical integration of the two-stage kinetic scheme we used for EPSCs (Compte et al., 2000) was excellent at all frequencies. $\boldsymbol{C}$. Histogram of the difference (error) between the measured and expected currents. Data from all three frequencies were combined in this histogram.

approximated by Ornstein-Uhlenbeck processes (Chance and Abbot, 2009).

Modeling synaptic background activity in this way, which has been called the "point conductance" method, requires integrating stochastic (rather than deterministic) differential equations and generating Gaussian random numbers. In our example code, we demonstrate how to do these things. Two conductance trains, one representing excitatory inputs and the other representing inhibitory inputs, were generated independently as OU processes. Each was determined by an equation of the form

$$
\frac{d g(t)}{d t}=-\frac{1}{\tau}\left[g(t)-g_{0}\right]+\sqrt{D} \chi(t),
$$

where $g(t)$ is the value of the conductance, $g_{0}$ is its mean value, $\tau$ is a time constant, $D$ is a diffusion constant, and $\chi(t)$ is a Gaussian white noise term of zero mean and unit standard deviation. As illustrated in Fig. $8 A$ (left), such an equation produces a random walk in time around the mean value, with a variance given by $\sigma^{2}=D \tau / 2$.

Introduction of this synaptic background activity had three distinct effects on the model cell (Fig. 8B): a depolarization of $5-10 \mathrm{mV}$, membrane potential fluctuations of $\sim 10 \mathrm{mV}$, and a decrease in input resistance of more than a factor of 5. Varying the mean and standard deviation of the excitatory and/or inhibitory conductances produced changes in total conductance and membrane potential that closely matched what would be predicted given the model cell parameters (Fig. $8 C$ ).

\section{Pyramidal neurons}

We further tested the system using whole-cell patchclamp recordings from layer $2 / 3$ and 5 pyramidal neurons in acute mouse prefrontal slices.

Layer $2 / 3$ pyramidal neurons have relatively little HCN current, at least when compared with pyramidal neurons in deeper layers (Biel et al., 2009). As a result, they re- 
A

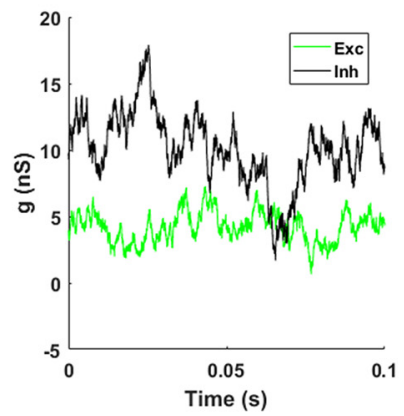

B

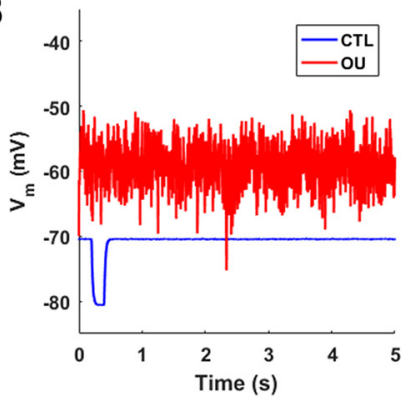

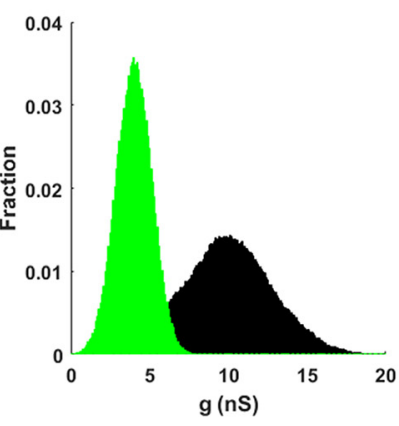

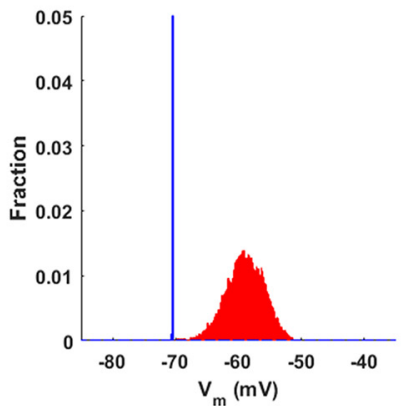

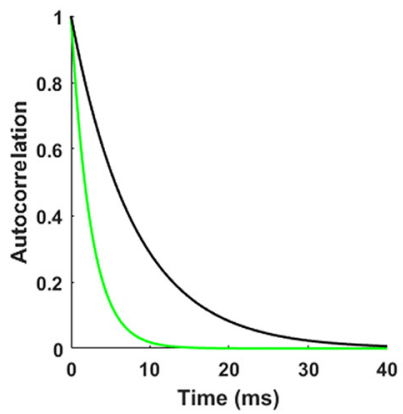

C

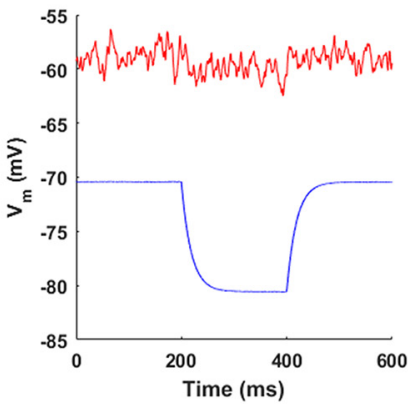

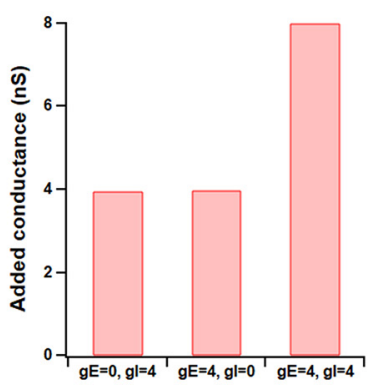

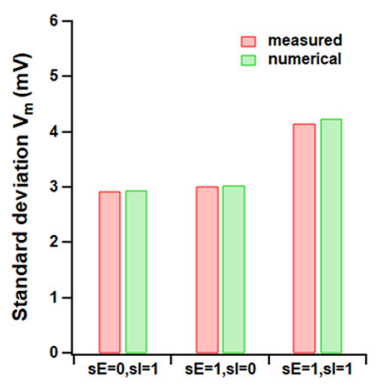

Figure 8. Synaptic background activity. $\boldsymbol{A}$, In the point conductance model of Destexhe et al., (2001), synaptic background activity is modeled by two noisy conductance trains. One represents excitatory input, and the other represents inhibitory input; both are generated by Ornstein-Uhlenbeck processes. Each train is normally distributed (middle) and is correlated at short times (right; the power spectrum goes like $1 / f^{2}$ for higher frequencies). $\boldsymbol{B}$, Without background activity, the model cell has a flat membrane potential (left) that is almost constant (middle); its input resistance is large (right). Adding in vivo-like background activity depolarizes the membrane, introduces membrane potential fluctuations, and reduces the input resistance. The vertical scale of the middle histogram is truncated so that the membrane potential distribution in the active state is easier to see. $\boldsymbol{C}$, Varying the mean ( $g$, in nanoSiemens) and standard deviation ( $s$, in nanoSiemens) of the excitatory and inhibitory conductances produced the predicted changes in total conductance (top) and membrane potential fluctuations (bottom). The added conductance is expected to equal the sum of $g E$ and gl. The numerical estimates of $V_{m}$ standard deviation were calculated by simulating the model cell. When $s E$ and $s /$ were varied, $g E$ and $g l$ were held fixed at $4 \mathrm{nS}$.

spond to temporally fluctuating input as low-pass filters, and they exhibit no or very small sag potentials. To test the first property, we injected a chirp stimulus (sinusoidal current with a frequency that increases in time, also known as a ZAP stimulus) into a layer $2 / 3$ pyramidal neuron. As expected, the voltage deflection dropped as the frequency increased (Fig. $9 A$, left). We quantified this effect by calculating the impedance, which can be thought of as a frequency-dependent resistance, by dividing the Fourier transforms of the voltage and the current (Fig. 9A, upper right). The impedance decreased monotonically between 0 and $15 \mathrm{~Hz}$. Into this neuron, we then introduced an HCN conductance of $6 \mathrm{nS}$ using the dynamic clamp system. With this addition, the voltage deflection in response to the chirp stimulus showed a peak (a resonance) when the stimulus frequency was near $5 \mathrm{~Hz}$ (Fig. 9A, left and upper right, traces in red). In the absence of the simulated HCN conductance, this neuron showed no sag potential, but addition of the HCN conductance both reduced the steady-state resistance (smaller voltage deflection in response to a fixed current) and added a sag potential (Fig. 9A, lower right).

To first order, the intrinsic excitability of neurons turns on the balance between hyperpolarizing currents (especially leak) and depolarizing currents (especially transient sodium). (To second and higher order, many other types of currents enter in.) We increased the intrinsic excitability of a layer $2 / 3$ pyramidal neuron by injecting 100 or 200 nS of simulated sodium conductance (Fig. 9B). A current step that was subthreshold in the basal state (left, trace in blue) produced one or six action potentials (left, traces in green and red) when sodium conductance amplitude was increased. Likewise, a current step that was just suprathreshold in the basal state (right) produced more and more spikes as sodium conductance amplitude was increased. Moreover, the latency to first spike and the threshold of the first spike dropped as sodium conductance was added.

In an acute slice, neurons exist in a quiescent state with spontaneous firing rates and $V_{m}$ fluctuations near zero. This is very different from the active state that exists in vivo (Destexhe et al., 2003). We simulated an in vivo-like active state in a layer 5 pyramidal neuron in a brain slice using the point conductance method (Fig. 9C). The neuron's activity reproduced the expected features of high conductance state background activity: a firing rate of 2 $\mathrm{Hz}$, a mean depolarization of $10 \mathrm{mV}$, and $V_{m}$ fluctuations with a standard deviation of $5 \mathrm{mV}$.

\section{Alternatives to Teensy}

We built the dynamic clamp system around the Teensy 3.6 microcontroller because of its speed and memory, but the approach is more general. It is not limited to this 


\section{A}
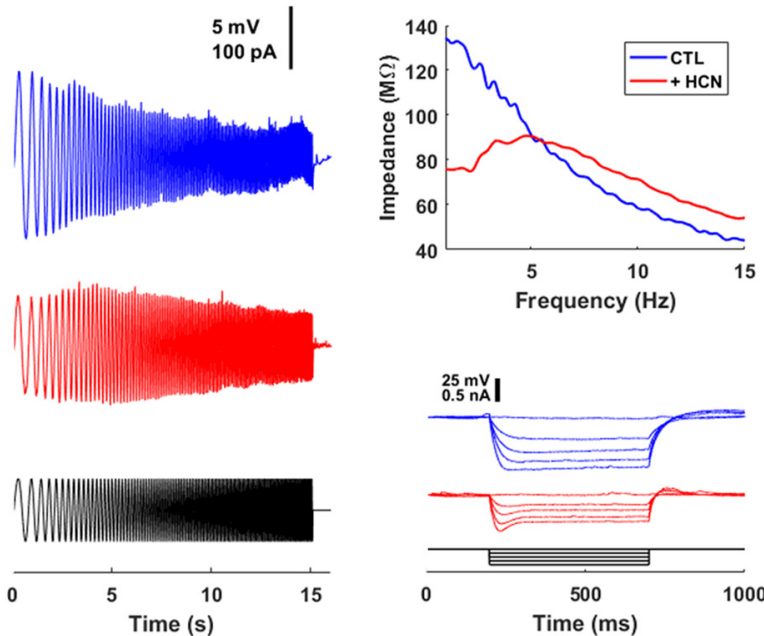

C

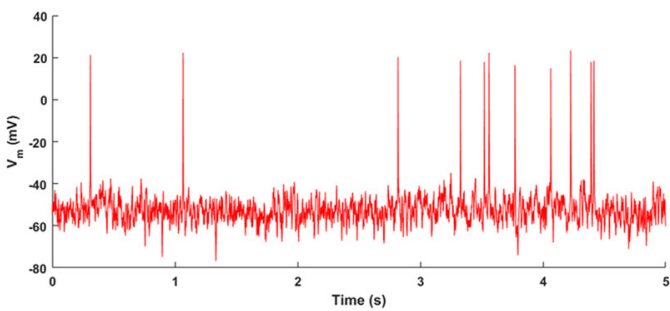

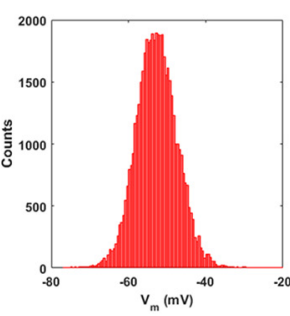

B
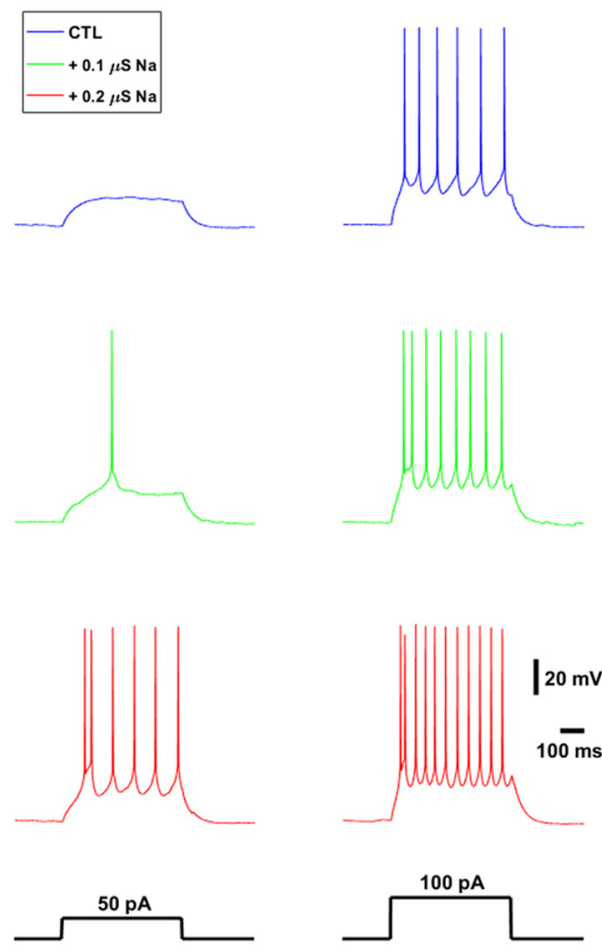

Figure 9. Dynamic clamp recordings from pyramidal neurons in slices of mouse prefrontal cortex. $\boldsymbol{A}$, HCN conductance. Left, a layer $2 / 3$ pyramidal neuron shows a low pass filtering response (blue) when subjected to a chirp current (black). This is transformed into a bandpass response (red) when $6 \mathrm{nS} \mathrm{HCN}$ conductance is added by the dynamic clamp. Upper right, the impedance profiles show this effect quantitatively. Lower right, the neuron also developed a sag potential in response to hyperpolarizing current injections. $\boldsymbol{B}$, Sodium conductance. Addition of 100 or 200 nS of sodium conductance makes this layer $2 / 3$ pyramidal neuron more excitable in a graded fashion. Shown are the responses to current steps of 50 and 100 pA. $\boldsymbol{C}$, Synaptic background activity in a layer 5 pyramidal neuron. The point conductance method was used to simulate an in vivo-like state (excitatory mean $3 \mathrm{nS}$, excitatory standard deviation $1.5 \mathrm{nS}$, inhibitory mean $6 \mathrm{nS}$, inhibitory standard deviation $3 \mathrm{nS}$ ). At left is a 5 -s recording of membrane potential $V_{m}$. At right is a histogram of subthreshold membrane potential; the spikes of the 5-s recording were clipped out, and the remainder were used to construct the histogram.

particular device. In fact, many other microcontrollers could be used with only slight modifications to the code. To underline this point, in the online material (folder Alternatives to Teensy) we include software and instructions for using an Arduino Due or a chipKit uC32 in place of the Teensy 3.6. The first is a member of the Arduino line of microcontrollers (www.arduino.cc); the second is based on a separate family (called PIC) of microcontrollers (chipkit.net).

\section{Discussion}

We have here introduced a dynamic clamp system built around a microcontroller and suitable for addition to any intracellular electrophysiology rig. In designing the system, we were guided by three requirements: (1) low cost (<USD\$100), (2) compatibility with the wide range of hardware and software found on existing rigs, and (3) accessibility to researchers with little prior experience with electronics or programming. The system not only meets these three requirements, but its performance is also comparable in accuracy and speed to those posted by the leading alternatives (Destexhe and Bal, 2009; Prinz and Cudmore, 2011). It was able to simulate with an average error of only a few percentage points the same variety of conductances other systems have been used to simulate, and its single-conductance speed $(90 \mathrm{kHz})$ was exceeded by only 3 of 24 published systems (pre-2012 systems reviewed by Lin [2012], Clausen et al. [2013], and Yang et al. [2015]).

The principal limitation on the accuracy of our system is that the ADC inputs and DAC outputs of the Teensy 3.6 microcontroller have 12-bit (4096-level) precision, rather than the 16-bit (65,536-level) precision available in recent $\mathrm{DAQ}$ systems. Although this is indeed a limitation, we would argue that it is not an important one. Consider the ADC input: 12-bit precision means that the input's 3.3-V total range is sampled in increments of $0.8 \mathrm{mV}$. Given the empirical relationship between ADC input and membrane potential $V_{m}$ (Fig. 3A), we were therefore able to measure $V_{m}$ with a precision of $0.06 \mathrm{mV}$. For physiologically realistic experiments, this is almost certainly good enough. And it is a lower limit: as we note in the online material ("Assembling the system"), one can adjust the resistor values to take better advantage of the ADC input's full dynamic range; our choices were conservative. An argument similar to the ADC one pertains to the DAC output. 
A different potential limitation on accuracy-one that our system shares with most existing dynamic clamp systems (Destexhe and Bal, 2009)-arises from our use of forward Euler numerical integration for the Hodgkin-Huxley and Ornstein-Uhlenbeck equations (Bettencourt et al., 2008). However, our simulation of the transient sodium conductance (Fig. 6) suggests that this is not a determinative limitation, because the microcontroller can do the integration with a very fast time step $(<12 \mu \mathrm{s})$, which is fast enough to obviate most problems. It is possible to implement a more precise numerical method on a Teensyclass microcontroller (see, e.g., http://bit.ly/2t6Jtyk), but this would come at the cost of simulation speed. For example, using the fourth-order Runge-Kutta method would drop the speed by a factor of two. There is a trade-off between accuracy and speed. Our data indicate that, even for transient sodium, forward Euler is satisfactory given how fast the Teensy microcontroller is.

The dynamic clamp system as a whole is fast because the microcontroller is doing nothing but implementing dynamic clamp routines. It is not maintaining an operating system, interacting with a user, updating a graphical interface, or implementing other unrelated routines. It is devoted exclusively to dynamic clamp. In this, it is similar to earlier hardware implementations, such as those based on digital signal processing boards (Desai and Walcott, 2006; Olypher et al., 2006), but with much reduced cost and complexity. Somewhat faster dynamic clamp systems have been introduced recently, including one based on Matlab's xPC Target software (Clausen et al., 2013; $>125 \mathrm{kHz}$ ) and another that uses Igor Pro software and National Instruments hardware (Yang et al., 2015; >100 $\mathrm{kHz}$ ), but, for many potential users, these would require substantial modification of existing electrophysiology systems, as well as the purchase of new hardware or software.

In principle, the dynamic clamp system we describe could be made faster. On every cycle, the system spends the bulk of its time doing two things: reading from the $A D C$ input and writing to the DAC output. One of our major goals was to keep the system as simple as possible, and so we used the built-in analog read and write functions of the Teensyduino version of the Arduino language. However, a defining feature of maker movement devices like the Teensy microcontroller-one that distinguishes them from many commercial devices-is that users have direct access to their inner workings; those innermost parts are not protected. One could reduce the time spent reading and writing the analog ports by addressing the registers directly and, for example, reducing how many samples the ADC input takes before reporting a result (see, e.g., https://github.com/pedvide/ADC). The cost of such a manipulation is in accuracy: it might increase noise. As always, there is trade-off between accuracy and speed. A smaller improvement might be obtained by using higher-quality electronic components. We chose the LM358 chip as an op-amp because of its ubiquity; it is available at nearly every hobbyist site. However, it has some limitations: it is not a "rail-to-rail" opamp, which means that its behavior becomes erratic if the input voltages get close to the $\pm 9 \mathrm{~V}$ rails of the power supply; it has a bandwidth and slew rate (how fast the voltage output can change in time) inferior to some other comparable op-amps (e.g., the OP484). Using these other op-amps would increase the cost of the system marginally ( USD\$20), but might be worthwhile depending on the user's needs and would not degrade performance in any case.

Other microcontrollers (such as the Arduino Due, the top-of-the-line Arduino model) could be used in place of the Teensy 3.6 with only small modifications, but we chose the Teensy for three reasons. It has a clock speed equal to or faster than its competitors (180 versus $84 \mathrm{MHz}$ for the Due), it has more memory (256 kB RAM versus 92 for the Due), and it has a floating point unit (which all of the Arduino models lack). The last point is the most important. Microcontrollers of the Arduino class tend to omit a floating point unit, a piece of hardware dedicated to and optimized for arithmetic on numbers where the number of digits after the decimal point might vary. (The opposite of a floating point number is called a fixed point number, which has a fixed number of digits after the decimal point. An integer is the simplest example.) Variables naturally represented by float point numbers are ubiquitous in neurophysiology and particularly in Hodgkin-Huxley calculations. One might attempt to transform floating point arithmetic to fixed point arithmetic for the sake of speed (instead of asking what $2.0 \times 2.00$ is, one might instead ask what $200 \times 200$ is and then divide the result by 10,000), but the Teensy's architecture obviates the need for such machinations. Again, it keeps things simple.

\section{References}

Anderson C. Makers: the new industrial revolution. New York: Crown, 2014.

Baden T, Chagas AM, Gage GJ, Marzullo TC, Prieto-Godino LL, Euler T (2015) Open Labware: 3-D printing your own lab equipment. PLoS Biol 13:e1002086. CrossRef Medline

Bettencourt JC, Lillis KP, White JA (2008) Effects of imperfect dynamic clamp: computational and experimental results. J Neurosci Methods 169:282-289. CrossRef Medline

Biel M, Wahl-Schott C, Michalakis S, Zong X (2009) Hyperpolarization-activated cation channels: from genes to function. Physiol Rev 89:847-885. CrossRef Medline

Biró I, Giugliano M (2015) A reconfigurable visual-programming library for real-time closed-loop cellular electrophysiology. Front Neuroinform 9:17 CrossRef

Chance FS, Abbott LF. Simulating in vivo background activity in a slice with the dynamic clamp. In: Dynamic-Clamp: From Principles to Applications. New York: Springer, 2009.

Clausen C, Valiunas V, Brink PR, Cohen IS (2013) MATLAB implementation of a dynamic clamp with bandwidth $>125 \mathrm{KHz}$ capable of generating INa at $37^{\circ} \mathrm{C}$. Pflugers Arch $465: 497-507$. CrossRef Medline

Compte A, Compte A, Brunel N, Goldman RPS, Wang XJ (2000) Synaptic mechanisms and network dynamics underlying spatial working memory in a cortical network model. Cereb Cortex 10: 910-923. CrossRef

Desai NS, Siegel JJ, Taylor W, Chitwood RA, Johnston D (2015) Matlab-based automated patch clamp system for awake behaving mice. J Neurophysiol 114:1331-1345. CrossRef Medline

Desai NS, Walcott EC (2006) Synaptic bombardment modulates muscarinic effects in forelimb motor cortex. J Neurosci 26:22152226. CrossRef Medline 
Destexhe A, Bal T (eds). Dynamic clamp: From principles to applications. New York: Springer, 2009.

Destexhe A, Mainen ZF, Sejnowski TJ (1994) Synthesis of models for excitable membranes, synaptic transmission and neuromodulation using a common kinetic framework. J Comput Neurosci 1:195230. CrossRef

Destexhe A, Rudolph M, Fellous JM, Sejnowski TJ (2001) Fluctuating synaptic conductances recreate in vivo-like activity in neocortical neurons. Neuroscience 107:13-24. Medline

Destexhe A, Rudolph M, Paré D (2003) The high conductance state of neocortical neurons in vivo. Nat Rev Neurosci 4:739-751. CrossRef Medline

Dorval AD, Christini DJ, White JA (2001) Real-time Linux dynamic clamp: a fast and flexible way to construct virtual ion channels in living cells. Ann Biomed Eng 29:897-907. Medline

Finley K. Out in the open: the men supercharging neuroscience with open source hardware. Wired March 10, 2014.

Fransen E, Alonso AA, Hasselmo ME (2002) Simulations of the role of the muscarinic-activated calcium-sensitive nonspecific cation current INCM in entorhinal neuronal activity during delayed matching tasks. J Neurosci 22:1081-1097. Medline

Gasparini S, Migliore M, Magee JC (2004) On the initiation and propagation of dendritic spikes in CA1 pyramidal neurons. J Neurosci 24:11046-11056. CrossRef Medline

Johnston D, Wu S. Foundation of cellular neurophysiology. Cambridge, MA: The MIT Press, 1994.

Kemenes I, Marra V, Crossley M, Samu D, Staras K, Kemenes G, Nowotny T (2011) Dynamic clamp with StdpC software. Nature Prot 6:405-417. CrossRef

Kullmann PHM, Wheeler DW, Beacom J, Horn JP (2004) Implementation of a fast 16-bit dynamic clamp using LabVIEW-RT. J Neurophysiol 91:542-554. CrossRef Medline

Lin, RJ. Real-time methods in neural electrophysiology to improve efficacy of dynamic clamp. Ph.D. dissertation, Georgia Institute of Technology, 2012.

Marsaglia G, Bray TA (1964) A convenient method for generating normal variables. SIAM Rev 6:260-264. CrossRef

Milescu LS, Yamanishi T, Ptak K, Mogri MZ, Smith JC (2008) Realtime kinetic modeling of voltage-gated ion channels using dynamic clamp. Biophys J 95:66-87. CrossRef Medline

Morozov E. Making it. The New Yorker January 13, 2014.

Narayanan R, Johnston D (2008) The h channel mediates locationdependence and plasticity of intrinsic phase response in rat hippocampal neurons. J Neurosci 28:5846-5860.

Nowotny T, Szucs A, Pinto RD, Selverston Al (2006) StdpC: a modern dynamic clamp. J Neurosci Methods 158:287-299. CrossRef Medline
Olypher A, Cymbalyuk G, Calabrese RL (2006) Hybrid systems analysis of the control of burst duration by low-voltage-activated calcium current in leech heart interneurons. J Neurophysiol 96: 2857-2867. CrossRef Medline

Ortega FA, Butera RJ, Christini DJ, White JA, Dorval AD (2014) Dynamic clamp in cardiac and neuronal systems using RTXI. Methods Mol Biol 1183:327-354. CrossRef Medline

Pinto RD, Elson RC, Szucs A, Rabinovich MI, Selverston Al, Abarbanel HDI (2001) Extended dynamic clamp: controlling up to four neurons using a single desktop computer and interface. J Neurosci Meth 108:39-48

Potter SM, El Hady A, Fetz EE (2014) Closed-loop neuroscience and neuroengineering. Front Neural Circuits 8:115. CrossRef Medline

Prinz AA, Abbott LF, Marder E (2004) The dynamic clamp comes of age. Trends Neurosci 27:218-224. CrossRef Medline

Prinz AA, Cudmore RH (2011) Dynamic clamp. Scholarpedia 6:1470. CrossRef.

Raikov I, Preyer AJ, Butera RJ (2004) MRCl: A flexible real-time dynamic clamp system for electrophysiology experiments. J Neurosci Meth 132:109-123. Medline

Robinson HPC, Kawai N (1993) Injection of digitally synthesized synaptic conductance transients to measure the integrative properties of neurons. J Neurosci Meth 49:157-165. CrossRef

Senturia SD, Wedlock BD. Electronic Circuits and Applications. New York: Wiley, 1975.

Sharp AA, Abbott LF, Marder E (1992) Artificial electrical synapses in oscillatory networks. J Neurophysiol 67:1691-1694. Medline

Sharp AA, O'Neil MB, Abbott LF, Marder E (1993) The dynamic clamp: artificial conductances in biological neurons. Trends Neurosci 16:389-394. Medline

Siegle JH, Cuevas López A, Patel Y, Abramov K, Ohayon S, Voigts J (2017) Open Ephys: an open-source, plugin-based platform for multichannel electrophysiology. J Neural Eng 14:045003.

Steriade, M. The intact and sliced brain. Cambridge, MA: MIT Press, 2001.

Teikari P, Najjar RP, Malkki H, Knoblauch K, Dumortier D, Gronfier C, Cooper HM (2012) An inexpensive Arduino-based LED stimulator system for vision research. J Neurosci Meth 211:227-236. CrossRef Medline

Walcott EC, Higgins EA, Desai NS (2011) Synaptic and intrinsic balancing during postnatal development in rat pups exposed to valproic acid in utero. J Neurosci 31:13097-13109. CrossRef Medline

Yang Y, Adowski T, Ramamurthy B, Neef A, Xu-Friedman MA (2015) High-speed dynamic-clamp interface. J Neurophysiol 113:27132720. CrossRef Medline 